\title{
Group structure and public goods provision in heterogeneous societies*
}

\author{
Jo Thori Lind ${ }^{\dagger}$ \\ Wednesday $24^{\text {th }}$ February, 2016
}

\begin{abstract}
I consider a society with heterogeneous individuals who can form organizations for the production of a differentiated public good. A decentralized arrangement of organizations is said to be split up stable whenever there is no majority to split any of the organizations. Compared to the social optimum, decentralization yields too few organizations if they provide broad services and potentially too many if they are highly specialized. Conclusions are broadly similar in the presence of an outside opportunity where only some individuals join organizations.
\end{abstract}

JEL codes: C62, D71, D72, H49, L31

Keywords: Organizations, public goods, split up stability, efficiency, endogeneous membership

*I am grateful for comments from two anonymous referees, an associate editor, Maren Elise Bachke, Bård Harstad, Tapas Kundu, Anthony McGann, Kalle Moene, and Fredrik Willumsen as well as seminar participants at the University of Oslo and at the EPCS and EEA conferences. While carrying out this research I have been associated with the center Equality, Social Organization, and Performance (ESOP) at the Department of Economics at the University of Oslo. ESOP is supported by the Research Council of Norway through its Centres of Excellence funding scheme, project number 179552.

$\dagger$ Department of Economics, University of Oslo, PB 1095 Blindern, 0317 Oslo, Norway. Email: j.t.lind@econ.uio.no. Tel. (+47) 22844027. 


\section{Introduction}

Organizations are everywhere in society, providing a huge range of services and ranging in scope from the local football club to nation-wide unions and international organizations. Most fields have a multitude of organizations providing comparable but still differentiated services. This is to accommodate the heterogeneous needs and tastes of their users. A large number of organizations guarantee each user access to services closely suited to her particular needs and desires. The flip side is higher total costs.

Do we get the appropriate number of organizations when they are allowed to form freely? In the celebrated papers by Salop (1979) and Alesina and Spolaore (1997), the answer is no. But this depends on how organizations form. I argue that under reasonable assumptions, we may actually achieve an optimal organizational structure in an unregulated economy.

My analysis applies to many categories of organizations. Economically, the most important may be the organization in the "third sector", the voluntary organizations providing welfare services complementing those provided by the public and private sectors. These organizations are responsible for providing health care, education, and many other services, and accounts for as much as $20 \%$ of GDP in some countries (Evers and Laville, 2004). Then efficiency matters. Most other organizations providing excludable public goods and services are also relevant examples, for instance churches (covering different denominations or religions), interest groups (advocating anything from stronger environmental protection to less strict gun regulation), newspapers (with differentiated focus and political orientation), sports and recreation facilities (for different sports, with different equipment, and at different location in space), housing cooperatives (different types of housing and location), and rotating savings groups (different income groups and risk profiles. Other applications are to the number of political parties (several differentiated parties may be required to get a properly functioning democracy) and the variety of unions (to accommodate different interests of workers in different sectors and different types of firms). In all of these cases, there are good reasons to entrust provision to private actors. But the efficiency of an unregulated market for organizations is an open question - there may be cases where the government can improve efficiency through subsidies or taxes.

I consider a population of heterogeneous individuals that can join organizations providing a unique public good. The utility derived from the public good depends on how well it matches the needs of the individual as well as on the cost of membership in the organization. In more heterogeneous societies where preferences are more diverse, a larger number of organizations is required to provide suitable services for everybody. Hence the socially optimal number of organizations is larger.

In the model, new organizations are formed by splitting old ones. If a majority of the members of an organization prefer a split, it splits. This can be seen as the organization holding a vote on whether to split, but a more natural interpretation is that a secessionist group leaves the organization if the support for maintaining unity is absent. ${ }^{1}$

As organizations are split and the number of organizations increases, support for further splits declines. This is both because organizations become smaller and hence closer to the needs of all its members, and because the financial burden on each member

\footnotetext{
${ }^{1}$ One could also imagine the center evicting the periphery, but in the class of models I consider this typically doesn't happen as the periphery pays membership fees and hence keep the costs down for individuals in the center.
} 
increases. When the splitting process has reached a state where only a minority of members in each organization prefers further split ups, the organizational structure is stable. I label this stable structure the split up stable equilibrium.

I go on to study the efficiency properties of decentralized equilibria where the number of organizations is given by the smallest split up stable number of organization. The welfare properties depend on the utility loss from an imperfect match between the member's ideal point and the public good provided by the organization. If this disutility is linear in distance, the members with median and average types coincide within each organization. As the median members are pivotal in the split up decision and the social optimum entails maximization of the utility of the member of average type, this implies that the split up stable outcome is almost efficient.

When preferences are concave in the distance from the ideal type, so organizations cater to a broader range of potential members, the median and average types no longer coincide. I show that in this case the decentralized solution yields fewer organizations and potentially too few organizations. Finally, if preferences are convex so organizations are specialized at providing services to a narrow segment of society, preferences for organizational splits become more involved as we get a coalition of the center and the remote periphery, both in favor of the old organizational structure, against the close periphery, who would be the winners in case of a split. The final outcome may be both too many and too few organizations.

Membership in organizations is voluntary in several interesting cases. Then it is unsatisfactory to require all individuals to belong to an organization. To model an enrollment decision, I give all agents an outside opportunity with heterogeneous value. Individuals with good outside opportunities stay outside the organizations, whereas individuals with bad outside opportunities join. This introduces a number of new features. First, the number of agents joining an organization depends on their types; agents who can find an organization close to their own preferences are more attracted to the organization, and join even when they have good outside opportunities. Among agents who are less satisfied with the organization's choice of public goods, only those with bad outside opportunities join. As it is now optimal that some agents, those with good outside options, stay outside the organizations, it follows that the optimal number of organizations is lower than with compulsory membership.

The main result from this extension is that with linear preferences, the smallest split up stable number of organizations still corresponds to the socially optimal number of organizations. There are three new factors pulling in different directions. First, with voluntary membership, agents whose ideal type of service is close to the one actually provided by the organization tend to be over represented within the organization as they are more likely to prefer the organization over the outside opportunity. These are less inclined to favor a split of the organization, reducing the pressure for splitting it. Second, as the cost of running the organization is split between the actual members, this also tends to limit the incentives to form new organizations. These two factors tend to give too few organizations. However, organizations are composed of agents with relatively bad outside opportunities, and when deciding on whether to spilt the organization or not, they do not take the preferences of agents with good outside opportunities into account. This third factor tends to give too many organizations. Under the conditions studied here, these factors almost perfectly balance, typically canceling out the effect of voluntary membership and hence restoring efficiency.

There are three major novelties in this paper: First, the split up stability criterion 
has not been used before. This solution concept is interesting because it is a reasonable criterion for the study of organization formation, because it provides a useful benchmark as the decentralized solution corresponds closely to the social optimum in certain cases, and because it by its transparency yields analytically tractable expressions. Second, the paper provides an analysis of how the decentralized solution compares to the social optimum under different assumptions on the cost of the difference between the agent's and the organization's type. Finally, this is to the best of my knowledge the first paper to consider the issue of endogeneous membership in this context.

The paper is related to several strands of literature. First, the formation of organizations may be seen as specific case of coalition formation, studied at length in cooperative game theory and related literature. It may also be seen as a special case of the literature on club goods, starting with Buchanan (1965); see e.g. Cornes and Sandler (1996), Scotchmer (2002), and Wooders (2012) for overviews. This literature focuses more on crowding and less on optimal diversification than the present work. Also, the dominant competitive approach of Ellickson et al. (1999) focuses on infinitely many small clubs whereas I consider a finite number of infinitely large organizations.

The modeling is closely related to Hotelling's (1929) model of choice of location, and the extensions by e.g. Salop (1979). But as this literature focus on competition and price mechanisms, both analyses and outcomes differ substantially. The paper is also closely related to Cremer et al.'s (1985) model of the location of facilities in space and the extensions and improvements thereupon by Fujita and Thisse (2002). However, their approach is based on a society-wide decision mechanism so no group can choose to form a new facility, and membership is compulsory. There are a large number of further contributions in this literature, including Casella and Feinstein (2002), Haimanko et al. (2004). Many of these consider more general frameworks and equilibrium concepts than I do. The advantage of the simple framework herein is that it is possible to go further in studying welfare properties of the equilibrium outcome than the more general framework allows.

The paper is maybe most closely related to a series of papers by Bogomolnaia et al. (2007, 2008a,b). They concentrate on models with linear transportation costs. In their 2007 and 2008b papers, they study stable organizational structures under various notions of stability and whether at all any stable structures exist. In their 2008a paper, they show that there exist stable structures with organizations of heterogeneous size under general conditions. Musatov et al. (2015) extend the proof of the existence of a stable structure to the case of non-uniformly distributed agents with an innovative use of the Gale-Nikaido-Debreu lemma.

There is also a large related literature on local public goods provision starting with Tiebout (1956). Parts of this literature, such as Westhoff (1977) and Jehiel and Scotchmer (1997), ask similar questions to the present paper. The focus is largely on the effect of mobility on equilibria in different jurisdictions whereas I use a type of heterogeneity where sorting into jurisdictions is not possible. Closer to the present paper is Wooders (1987; 1989), but she restricts her attention to a finite number of types. In political science, there is also a small literature studying multi-party systems in a similar fashion (McGann, 2002), but this literature pays little attention to the determination of the number of parties. The paper is also related to the literature on the size and number of nations (Bolton and Roland, 1997; Alesina and Spolaore, 1997, 2003; Le Breton and Weber, 2003; Haimanko et al., 2005) and formation of international unions (Ruta, 2005). There are some major differences between the formation of countries and other organizations, 
though. First, countries are typically formed by the unification of tribes into chiefdoms and further into centralized states ${ }^{2}$ whereas organizations tend to arise from one or a few initial organizations through repeated secession processes. Then the natural criteria for stability differ. Secession is a prominent question in many contemporary countries too, but potential secessionists typically face stronger opposition from the central power than does a secessionist faction in an organization - secession from countries is typically against the constitution whereas the right to form new organizations is assured by the same constitution. Finally, as membership in countries is not voluntary, there is no question of endogeneous membership in this literature.

Jehiel and Scotchmer (2001) consider the converse to my problem, the formation of jurisdictions where entrants can be denied. There is also a literature on the location and size of cities (Krugman, 1993; Tabuchi et al., 2005), but this literature, although conceptually close, is quite different in the way the economy and the set of possible locations is modeled. Finally, the paper is related to the literature on group formation (e.g. Milchtaich and Winter, 2002), and the literature on private provision of public goods (Bergstrom et al., 1986). This literature is more centered on the problem of crowding, which I disregard. Also, stability is not considered, as they abstract from the threat of secession.

\section{The model}

Organizations provide services of different varieties, where a variety is some point on the unit interval $[0,1] .{ }^{3,4}$ There is a total of $N$ organizations, where the determination of $N$ is the objective of the paper. Each of these provides some variety $q_{i} \in[0,1]$. The point $q_{i}$ is referred to as the organization's type.

There is a continuum of agents, where agents' preferences are uniformly distributed on $[0,1]$. An agent's location $x$ on the unit interval describes her preferences for the services provided by the organizations - the closer the organization is to $x$, the better suited its services are. The position $x$ is referred to as the agent's type.

Initially, I assume that all individuals are members of an organization. ${ }^{5}$ This is relaxed in Section 4. Membership in multiple organizations would not serve any purpose in the current framework, and is disregarded. See e.g. Weber et al. (2014) for an analysis of cases where it does matter.

An agent of type $x$ derives utility $u\left(\left|x-q_{i}\right|\right)$ from joining organization $i$, where $u$ is a decreasing function and $\left|x-q_{i}\right|$ is a measure of the dissimilarity between the agent's tastes and the services provided by the organization. The slope of $u$ can be interpreted as a measure of the degree of heterogeneity in society: In heterogeneous societies tastes are very different across individuals so the utility of organization membership depends crucially on its services being close to own tastes, whereas they are more comparable in more homogeneous societies. If the utility function $u$ is convex it indicates a specialized organization that suits a small group and most others are less well served. A concave function indicates a broader organization where a quite large group is quite well served.

\footnotetext{
${ }^{2}$ See e.g. Fukuyama (2011) for an overview of theories of these processes.

${ }^{3}$ To keep the model tractable, I focus on one dimensional heterogeneity among organizations and members. A common finding in this class of models is that with general distributions and multi-dimensional heterogeneity, one can under some conditions show the existence of a stable partitioning of agents (Drèze et al., 2008), but few other robust results exist (McGann, 2002).

${ }^{4}$ Similar results could be obtained by considering varieties on a circle, instead of a line.

${ }^{5}$ Equivalently, the outside option of not joining any organization yields utility $-\infty$.
} 
There is a fixed cost $C$ of running an organization, independently of its size. ${ }^{6}$ These costs are covered by a uniform membership fee $c$ on each member. When an organization attracts members from a set $S$, the mass of members is $\|S\|$ and we have $c=\frac{C}{\|S\|}$. Below we see that all organizations become equally sized so $\|S\|=\frac{1}{N}$ and $c=N C$. If we measure utility in monetary terms, a member of type $x \in[0,1]$, belonging to an organization $i$ of type $q_{i}$ and paying a membership fee $c$, derives total utility

$$
U\left(x, q_{i}\right)=u\left(\left|x-q_{i}\right|\right)-c
$$

\subsection{The social optimum}

The social optimum is found as the optimal trade off between higher costs of more organizations and higher average dissatisfaction of organizational type. The social planner's objective is to choose a number of organizations $N$ and the locations $\left\{q_{i}\right\}_{i=1}^{N}$ of these to maximize

$$
\int_{0}^{1} u(|x-q(x)|) d x-N C
$$

where $q(x)=\arg \min _{\tilde{q} \in\left\{q_{i}\right\}}|x-\tilde{q}|$ is the optimal organization to join for an agent of type $x$. The optimal choice of $N$ and $\left\{q_{i}\right\}_{i=1}^{N}$ is simultaneous, but as it is easy to find the optimal locations $\left\{q_{i}\right\}_{i=1}^{N}$ for any value of $N$ I solve the problem in two steps. Specifically, with $N$ organizations, it is optimal to have an equal spacing of organizations. Formally we have

Proposition 1. In a social optimum with $N$ organizations, the provided types are

$$
q_{i}=\frac{2 i-1}{2 N}, i=1, \ldots, N .
$$

Proof. Define the function $V(r)=2 \int_{0}^{r} u(x) d x$. For any decreasing $u, V$ is a concave function. The social planner's objective is then identical to choosing a partition of the unit interval into $N$ segments of length $R_{1}, \ldots, R_{N}$ to maximize $\sum_{i=1}^{N} V\left(R_{i}\right)$. Given concavity and an interior solution, maximizing $\sum_{i=1}^{N} V\left(R_{i}\right)$ subject to $\sum_{i=1}^{N} R_{i}=1$ yields for any $i, j$ that $V^{\prime}\left(R_{i}\right)=V^{\prime}\left(R_{j}\right)$, i.e. $u\left(R_{i}\right)=u\left(R_{j}\right)$. As $u$ is monotonic, it follows that the optimal choice of $R_{i}$ is a partition into segments of equal length.

The intuition for this result is simply that the optimal location of organization is an even distribution so the average distance is minimized. As we have equally sized organizations in optimum, the social planner's objective (1) can be rewritten as

$$
W(N)=2 N \int_{0}^{\frac{1}{2 N}} u(x) d x-N C .
$$

As it is only meaningful to consider an integer number of organizations, ordinary maximization of $W$ is not meaningful. However, it is easily seen that the optimal number of organizations is (weakly) decreasing in costs $C$. Hence it suffices to study the cost levels where $N$ and $N+1$ organizations give the same social welfare; call this level $S_{N}$. The values $\left\{S_{N}\right\}_{N=0}^{\infty}$ provides a partitioning of the possible cost levels such that the social

\footnotetext{
${ }^{6} \mathrm{~A}$ relaxation of the fixed cost assumption is discussed in Section 2.3.
} 
planner chooses $N$ organizations iff $C \in\left[S_{N}, S_{N-1}\right){ }^{7}$ By definition, $S_{0}=+\infty$. We see that $W(N)=W(N+1)$ implies

$$
2 N \int_{0}^{\frac{1}{2 N}} u(x) d x-N S_{N}=2(N+1) \int_{0}^{\frac{1}{2 N+2}} u(x) d x-(N+1) S_{N},
$$

so for $N \geq 1$ we get

$$
\begin{aligned}
S_{N} & =2(N+1) \int_{0}^{\frac{1}{2 N+2}} u(x) d x-2 N \int_{0}^{\frac{1}{2 N}} u(x) d x \\
& =2 \int_{0}^{\frac{1}{2 N+2}} u(x) d x-2 N \int_{\frac{1}{2 N+2}}^{\frac{1}{2 N}} u(x) d x .
\end{aligned}
$$

The right hand side of the expression gives the increased welfare of an additional organization. This main effect is the increased welfare of a new organization (the first part of the expression). A social planner should, however, also take into account that the members of this organization does not come from nowhere but instead from the tails of the pre-existing organizations (the second part of the expression). This effect is closely related to an external effect, and we see below that it is indeed ignored in the decentralized solution.

\subsection{The decentralized solution: The split up stable outcome}

The next step is to see how organizations are created and of which types they are when they are controlled by their members. I focus on a process where any member of an organization can propose to split the organization into two separate organizations. The decision on whether to split is made as if it was determined by majority voting. We may also think of this as the factions in the periphery of the organization uniting to force the center to accept a split without a vote actually taking place. If the configuration of organizations is such that there is no organization with a majority for splitting it, I label the configuration split up stable.

Definition 1. A number of organizations is split up stable if no organization contains a majority for splitting it into two organizations.

Throughout, I look at situations where agents can choose which organization to join.

One key point in determining split up stability is the agents' expectations of the effects of a split. Throughout most of the paper, I focus on an approach where the agents compare the initial organization to a situation where it is split in two equal parts. This is probably a quite accurate description of the debate that would take place when considering whether to split. A split in one organization may eventually lead to changes in the composition of members in other parts of the organizational spectrum, but this would usually be ignored. If the organization for the protection of the whales and the organization for the protection of the seals were to split up, the effect on the membership in the gun lobby would not be center stage in their debate. One may argue that this approach is too myopic. An organization may after all hope to take some members from

\footnotetext{
${ }^{7}$ When $C=S_{N}$ the social planner is indifferent between $N$ and $N+1$ organizations. To achieve a complete partitioning of the real line, I assume that he the prefers $N$ organizations.
} 
neighboring organizations in case of a split. A partial outlook is difficult to model in a rigorous way, but I study the perfectly global view in Section 3.2.

The relationship between the split up stable outcome and some other equilibrium concepts found in the literature is discussed further in Section 3. Split up stability implies that there is no incentive to split the organization, in the sense that the majority of members in the organization prefers to keep it intact. If on the contrary there is such a majority, a split could be achieved by a vote within the organization. The possibility of factions unilaterally leaving the organization is also discussed in Section 3 - generally this is difficult when a majority opposes a split.

It is easily seen that for sufficiently many organizations, split up stability always holds. More interesting is to study the smallest number of organizations we can have and still maintain split up stability. The equilibrium in the decentralized solution is taken to be the smallest number of organizations that satisfy split up stability. One way to think about this equilibrium is to start with one organization. If there is a majority to split it, it splits, otherwise $N=1$ is stable. With two organizations, we next have to check whether each of these are stable. If they are not, one of them splits and members are redistributed evenly among the three new organizations. The process continues until we reach stability.

More generally, consider the following sequence of games to determine the number of organizations, the type of each organization, and which agents belong to each organization: Initially, society starts with $N_{0}$ organizations, ${ }^{8}$ and each of these organizations have some exogeneously determined location $\left\{q_{i}^{0}\right\}_{i=1}^{N_{0}}$. We then go through the following steps:

1. Individuals are allocated to organizations to from equally sized organizations ${ }^{9}$

2. Each organization decides its type by majority voting

3. Each agent can choose to switch to another organization. If anybody does, we go back to step 2

4. In each organization, members can propose a split. If a split gets a majority within the organization, it is split in two organizations and we move back to step 1

Whenever we reach step 4 without a split getting a majority in any organization, we are at an equilibrium of the game.

As the organization's type is a unidimensional decision and preferences over types are single peaked, the median voter theorem applies. Hence the chosen type at step 2. corresponds to its center, which is also the socially optimal location. The final point to analyze is the split up decisions at step 4, where the notion of split up stability comes in.

\subsubsection{The weakly concave case}

Consider first the case where $u$ is linear or concave. It turns out that the analysis of a convex $u$ function is somewhat more involved, so this is postponed until Section 2.2.2. Consider without loss of generality the first organization, which covers the interval $\left[0, \frac{1}{N}\right]$.

\footnotetext{
${ }^{8} \mathrm{I}$ assume that $N_{0}$ is sufficiently small that this is a question of organization formation. The question of organizational merger is discussed in Section 3.3.

${ }^{9}$ This can typically be achieved by individuals freely choosing which organization to join. However, as shown by Bogomolnaia et al. (2008a), there are (unstable) equilibria with heterogeneous organization size. It is difficult to completely rule out the process getting stuck in one of these.
} 
The membership fee is $c=C N$, and by the median voter theorem, the organization is of type $\frac{1}{2 N}$. If the organization splits in two equal parts, the new membership fee becomes $2 C N$, and the type becomes $\frac{1}{4 N}$ and $\frac{3}{4 N}$. The distribution of preferences between splitting and not is shown in Figure 1.

If the members of types $\frac{1}{4 N}$ and $\frac{3}{4 N}$ favor a split, then so will the members of types $x<\frac{1}{4 N}$ and $x>\frac{3}{4 N}$ whenever $u$ is weakly concave. In the linear case, all these members enjoy the same gain from an organizational split. In the case of a concave $u$, the gains from the split are smallest for the agents located at $\frac{1}{4 N}$ and $\frac{3}{4 N}$, and increasing the closer one get to 0 or $\frac{1}{N}$. This groups of agents constitute a majority within the organization.

If on the contrary the members of types $\frac{1}{4 N}$ and $\frac{3}{4 N}$ oppose a split, then so will the members of types $\left(\frac{1}{4 N}, \frac{3}{4 N}\right)$. These also constitute a majority. Hence the decision favored by the members of types $\frac{1}{4 N}$ and $\frac{3}{4 N}$ (which are coinciding) is the Condorcet winner. Consequently, $N$ organizations is split up stable if these members (weakly) prefer no split to a split. This is the case when

$$
u\left(\frac{1}{4 N}\right)-C N \geq u(0)-2 C N .
$$

Hence there is a critical level $D_{N}$ defined by

$$
D_{N}=\frac{1}{N} u(0)-\frac{1}{N} u\left(\frac{1}{4 N}\right)
$$

such that $N$ organizations is a split up stable configuration if $C \geq D_{N}$. Notice that this critical level can be seen as twice the area of a rectangle with height $u(0)-u\left(\frac{1}{4 N}\right)$ and width $\frac{1}{2 N}$, the rectangle shown in Figure 2 .

We saw above that a social planner chooses $N$ organizations whenever $C \in\left[S_{N}, S_{N-1}\right)$. Also, a number $N$ of organizations is split up stable whenever $C \geq D_{N}$ so $N$ is the smallest split up stable number of organizations when $C \in\left[D_{N}, D_{N-1}\right)$. Hence to study whether the decentralized equilibrium is socially optimal or not is equivalent to determining whether $S_{N}$ corresponds to $D_{N}$ or not. If $S_{N}<D_{N}$, there are cost levels with over provision of organizations, and vice versa. ${ }^{10}$

Consider first the case when the disutility of joining an organization different from the preferred type is linear so the utility function can be written $u(x)=1-a x$ for some constant $a>0$. We get $S_{N}=\frac{a}{4 N(N+1)}$. In a decentralized solution with linear disutility, $N$ organizations is split up stable when the cost is above $D_{N}=\frac{a}{4 N^{2}}$. As $S_{N}<D_{N}$, there is a tendency for over provision of organizations in the decentralized case, in the sense that there are values of $C$ so that the decentralized solution yields more organizations than the social optimum. However, as $D_{N+1}<S_{N}, N+1$ organizations is always split up stable when the social optimum is $N$ organizations.

Proposition 2. When preferences are linear in distance and the social optimum is $N$ organizations, $N+1$ organizations are always split up stable. There are also cases where $N$ organizations is split up stable.

To understand this near optimality result, notice that with linear preferences, the pivotal median member is also a member with average type. Consequently, the preferred outcome of the median members correspond to the average preference of the organization

\footnotetext{
${ }^{10}$ For a given preference structure $u$, we may have $S_{N}<D_{N}$ and $S_{N^{\prime}}>D_{N^{\prime}}$ for $N \neq N^{\prime}$. It is even possible to construct cases where $\left[D_{N}, D_{N-1}\right] \subset\left[S_{N}, S_{N-1}\right]$ and cases where $\left[S_{N}, S_{N-1}\right] \subset\left[D_{N}, D_{N-1}\right]$.
} 
Figure 1: Utility from splitting up (dashed line) and not splitting up (solid line)

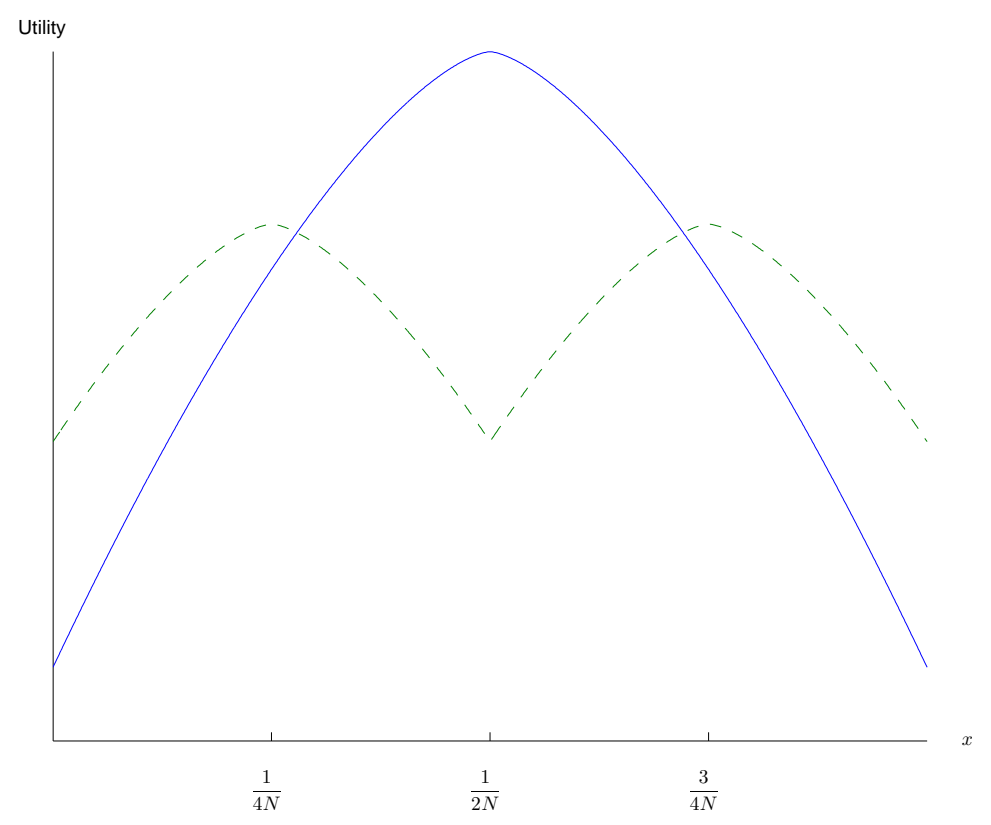

members. From a social planner's point of view, this is the utility of an additional organization disregarding the external effect, i.e. the first but not the second integral of equation (3). To see this graphically, consider Panel (a) of Figure 2. With linear costs, the rectangle corresponding to $D_{N}$ has an area exactly equal to the triangle corresponding to $2 \int_{0}^{\frac{1}{2 N+2}} u(x) d x$, i.e. the first part of equation (3). However, $S_{N}$ is slightly lower than $D_{N}$ because we have to subtract the second part of (3), $2 N \int_{\frac{1}{2 N+2}}^{\frac{1}{2 N}} u(x) d x$, which correspond to the $N$ small triangles in Figure $2{ }^{11}$ If we could disregard the latter effect, split up stability would coincide exactly with the social optimum under linear preferences. The reason for this is that the utility loss in going from a distance of 0 to a distance of $\frac{1}{4 N}$ exactly correspond to the average loss in going from 0 to the distances in $\left(0, \frac{1}{2 N}\right)$, which is what the social planner takes into account. This consideration only includes the increased utility of an extra organization, not the dampening effect from these members already coming from an organization. This effect is the reason why one organization above the social optimum may be required for split up stability. However, this effect is sufficiently small that there can only be one excess organization with linear preferences.

Consider now the case with more general preferences. Figure 2 shows $S_{N}$ and $D_{N}$ for some number $N$ in the case of linear, concave, and convex preferences. As seen in Section 2.2.2, the rectangle specification of $D_{N}$ conveys less interesting information in the convex case, though.

Concave preferences (Panel (b) in Figure 2) represent the case where organizations are able to cater to quite broad groups. In this case, a too low number of organizations may be split up stable:

\footnotetext{
${ }^{11}$ One could also see $S_{N}$ as (twice) the area of the triangle $2 \int_{0}^{\frac{1}{2 N}} u(x) d x$ minus $N+1$ of the smaller triangles $2 N \int_{\frac{1}{2 N+2}}^{\frac{1}{2 N}} u(x) d x$.
} 
Proposition 3. When $u$ is concave, $S_{N}-D_{N}$ is larger than under its linear counterpart $u_{\ell}(x)=u(0)+x u\left(\frac{1}{2 N}\right)$.

Proof. We compare preferences given by $u$ to a linear version given by $\tilde{u}(x)=u(0)-$ $2 N\left[u(0)-u\left(\frac{1}{2 N}\right)\right] x$. The proof is in two steps; I first show that the proposition holds for a special class of utility functions, and then that it holds for all concave preferences if it holds for this class.

1) Consider the class of utility functions

$$
u_{\gamma}(x)= \begin{cases}u(0)-2 N(1-\gamma)\left[u(0)-u\left(\frac{1}{2 N}\right)\right] x & \text { if } x<\frac{1}{2 N} \\ u\left(\frac{1}{2 N}\right) & \text { if } x=\frac{1}{2 N}\end{cases}
$$

for some $\gamma \in[0,1]$. At $\gamma=0$ the utility function corresponds to $\tilde{u}$. As this function has a discontinuity at $\frac{1}{2 N}$ it is not a concave function. However, it is the limit of a sequence of more and more concave functions.

The socially optimal cut offs for $u_{\gamma}$ are

$$
S_{N}^{\gamma}=2 \int_{0}^{\frac{1}{2 N+2}} u_{\gamma}(x) d x-2 N \int_{\frac{1}{2 N+2}}^{\frac{1}{2 N}} u_{\gamma}(x) d x=\frac{1-\gamma}{2(N+1)}\left[u(0)-u\left(\frac{1}{2 N}\right)\right]
$$

The corresponding cut offs for split up stability are

$$
D_{N}^{\gamma}=\frac{u_{\gamma}(0)-u_{\gamma}\left(\frac{1}{4 N}\right)}{N}=\frac{1-\gamma}{2 N}\left[u(0)-u\left(\frac{1}{2 N}\right)\right]
$$

It is easily seen that so for all $\gamma \in[0,1], S_{N}^{\gamma}<D_{N}^{\gamma}$ and moreover that $S_{N}^{\gamma}-D_{N}^{\gamma}$ is increasing in $\gamma$ whenever $\gamma \in[0,1]$. This proves the Proposition for preferences described by $u_{\gamma}$.

2) For any concave utility function $u$, take $\hat{\gamma}=1-\frac{N+1}{N} \frac{u(0)-u\left(\frac{1}{2 N+2}\right)}{u(0)-u\left(\frac{1}{2 N}\right)}$ so $u\left(\frac{1}{2 N+2}\right)=$ $u_{\hat{\gamma}}\left(\frac{1}{2 N+2}\right)$. On $\left[0, \frac{1}{2 N+2}\right]$ the linearized form $u_{\hat{\gamma}}$ forms an arc to $u$ so from concavity, $u(x) \geq u_{\hat{\gamma}}(x)$ for all $x$ in this interval. Hence

$$
\int_{0}^{\frac{1}{2 N+2}} u(x) d x \geq \int_{0}^{\frac{1}{2 N+2}} u_{\hat{\gamma}}(x) d x
$$

Furthermore, for a small $\epsilon>0$ we have $u\left(\frac{1}{2 N+2}-\epsilon\right) \geq u_{\hat{\gamma}}\left(\frac{1}{2 N+2}-\epsilon\right)$ so $u^{\prime}\left(\frac{1}{2 N+2}\right) \leq$ $u_{\hat{\gamma}}^{\prime}\left(\frac{1}{2 N+2}\right) \leq 0$. For all $x \in\left[\frac{1}{2 N+2} \frac{1}{2 N}\right)$ we have $u^{\prime \prime}(x) \leq 0$ by concavity and $u_{\hat{\gamma}}^{\prime \prime}(x)=0$ by assumption. It follows that

$$
\int_{\frac{1}{2 N+2}}^{\frac{1}{2 N}} u(x) d x \leq \int_{\frac{1}{2 N+2}}^{\frac{1}{2 N}} u_{\hat{\gamma}}(x) d x
$$

and hence that $S_{N} \geq S_{N}^{\hat{\gamma}}$.

Finally, as $u(x) \geq u_{\hat{\gamma}}(x)$ for all $x \in\left[0, \frac{1}{2 N+2}\right]$, we have $u\left(\frac{1}{4 N}\right) \geq u_{\hat{\gamma}}\left(\frac{1}{4 N}\right)$ for all $N \geq 1$, and hence $D_{N} \leq D_{N}^{\hat{\gamma}}$. Hence the Proposition holds for all concave preferences.

It follows that with concave preferences, we can get both over and under provision, but in the case of over provision, the tendency is weaker than with linear preferences. 

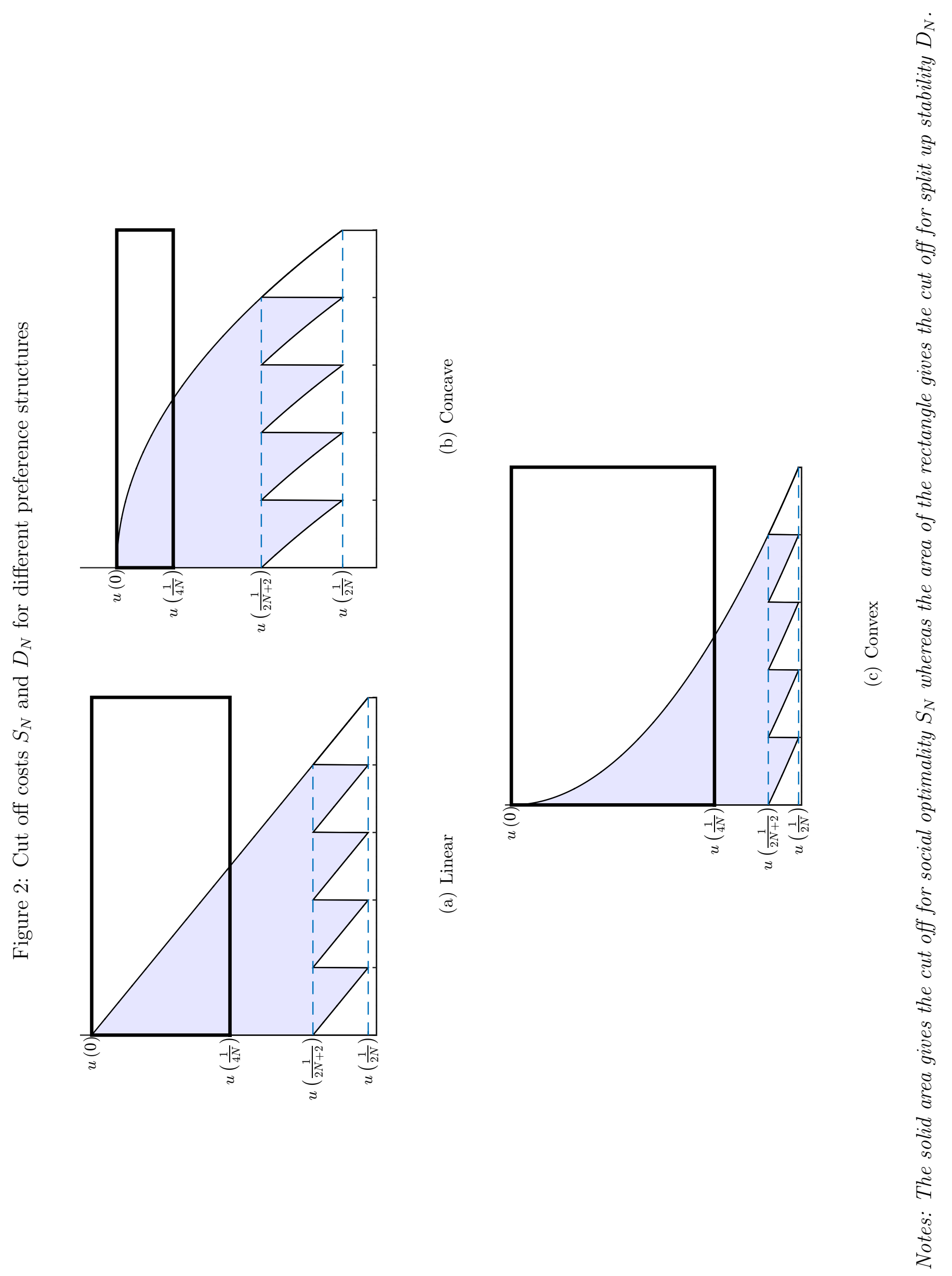
Moreover, it follows that there are also concave preferences where the social optimum corresponds exactly to split up stability. The split up stable outcome corresponds to the social optimum when the preference structure has a utility function $u$ which satisfies

$$
2 \int_{0}^{\frac{1}{2 N+2}} u(x) d x-2 N \int_{\frac{1}{2 N+2}}^{\frac{1}{2 N}} u(x) d x=\frac{u(0)-u\left(\frac{1}{4 N}\right)}{N}
$$

For a given number of organizations $N$, there is a large class of preferences where (6) is satisfied. If the utility function takes the form $u(x)=1-a x^{\kappa}$, we have $S_{N}^{\kappa}=$ $\frac{a}{2^{\kappa}(\kappa+1)}\left(\left(\frac{1}{N}\right)^{\kappa}-\left(\frac{1}{N+1}\right)^{\kappa}\right)$ and $D_{N}^{\kappa}=\frac{a}{4^{\kappa}}\left(\frac{1}{N}\right)^{1+\kappa}$. Hence the two coincides if $\kappa$ solves $N-\frac{N^{\kappa+1}}{(N+1)^{\kappa}}=\frac{\kappa+1}{2^{\kappa}}$. For $N=1$, the two coincides for $\kappa=2$, whereas they coincide for $\kappa=1$ as $N \rightarrow+\infty$.

For (6) to be satisfied for all $N$, the equation has to be seen as an integral equation that the utility function $u$ has to solve. However, it is only meaningful that the equation holds for integer $N$, which allows a larger class of functions to satisfy it. It is for instance possible to construct piecewise linear functions that satisfy the equation.

\subsubsection{The convex case}

Convex preferences mean that organizations are more specialized toward one specific type of members. The maximum cost from a social point of view, the shaded area in Figure 2 , typically shrinks. Due to the external effect, however, there are cases where it does not. At the same time, the maximum cost for split up stability, shown as the rectangle, increases. This effect always dominates the reverse effect of the externality. Hence when we compare the split up stable outcome with the one achieved with preferences $\tilde{u}(x)=$ $u(0)-2 N\left[u(0)-u\left(\frac{1}{2 N}\right)\right] x, S_{N}$ is lower in the case of convex preferences so the optimal number of organizations is weakly lower. The proof is provided in Appendix A.1 and is largely a converse to the proof of Proposition 3.

With convex preferences, there are two groups centered around $\frac{1}{4 N}$ and $\frac{3}{4 N}$ that are eager to split the organization. If these groups were to dictate, we would get over provision of organizations. However, unlike the weakly concave case considered above, the gains from such a split are largest for agents close to $\frac{1}{4 N}$ and $\frac{3}{4 N}$ and decreasing when one moves closer to 0 and $\frac{1}{N}$. This means that support from the agents located at $\frac{1}{4 N}$ and $\frac{3}{4 N}$ is now insufficient to assure a majority for a split.

To find the cost levels $D_{N}$ that assure a majority for a split, we need to find the fraction of agents in favor of a split for any cost level $C$. Denote by $\underline{i}$ and $\bar{i}$ the two members that are indifferent between a split and no split. Then $D_{N}$ can be derived from the following system of equations:

$$
\left\{\begin{aligned}
u\left(\frac{1}{4 N}-\underline{i}\right)-D_{N} & =u\left(\frac{1}{2 N}-\underline{i}\right) \\
u\left(\bar{i}-\frac{1}{4 N}\right)-D_{N} & =u\left(\frac{1}{2 N}-\bar{i}\right) \\
\bar{i}-\underline{i} & =\frac{1}{4 N}
\end{aligned}\right.
$$

As both $S_{N}$ and $D_{N}$ are lower under convex preferences it is unclear whether we get over or under provision of organizations. As a matter of fact, both cases are possible. To 
Figure 3: $S_{N}$ and $D_{N}$ in a parameterized model

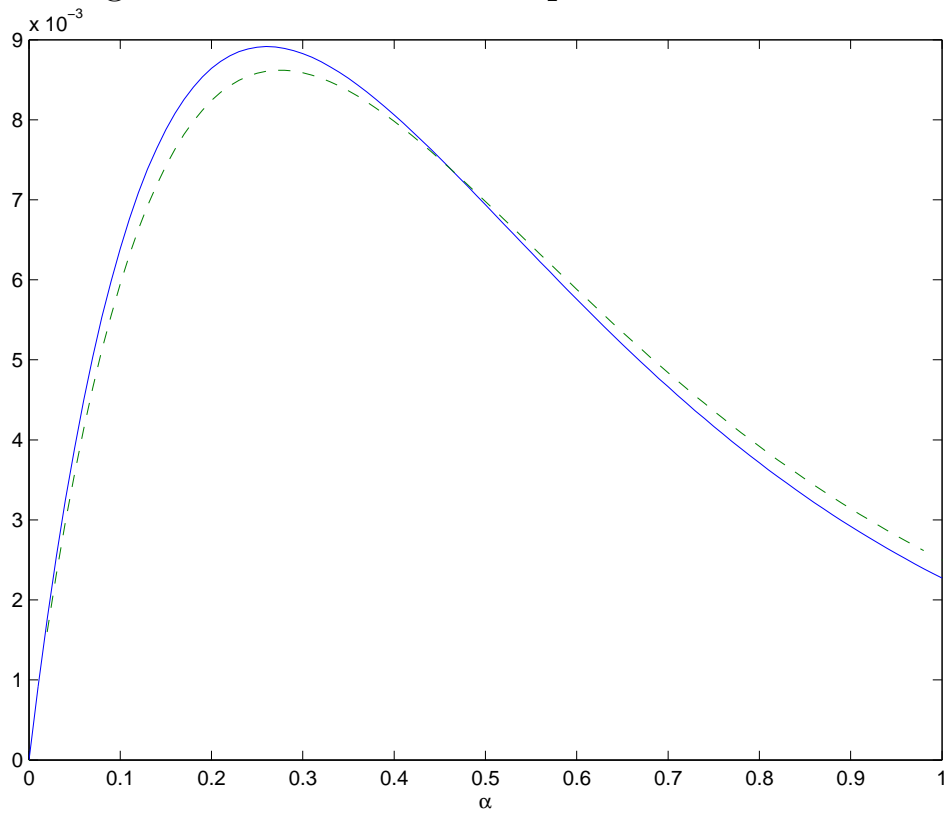

Notes: The figure shows $S_{N}$ (solid blue lines) and $D_{N}$ (dashed green lines) for $N=10$ and different values of $\alpha$ with the specification $u(x)=1-x^{\alpha}$.

show this, consider the specific case where $u(x)=1-x^{\alpha}$. When $N$ is 1 or 2 , we have $D_{N}>S_{N}$ for all values of $\alpha>0$. But for $N \geq 3$ we have $D_{N}<S_{N}$ for low values of $\alpha$ and $D_{N}>S_{N}$ for high values. Figure 3 shows the case with $N=10$ where we have a crossing at about $\alpha \approx 0.47$.

\subsection{Variable costs}

The model discussed above assumes that each organization has a fixed cost of operating. Usually, it is more costly to run larger organizations so there is a variable component in addition to the fixed costs. Generally we could have a cost function $C\left(\frac{1}{N}\right)$. However, we could still use the approach developed above albeit with a few modifications. First, the smallest split up stable number of organizations is the smallest $N$ such that $C\left(\frac{1}{N}\right) \geq D_{N}$. Second, a number of organizations $N$ is socially optimal if $S_{N} \geq C\left(\frac{1}{N}\right)>S_{N-1}$. However, this criterion does not always give a unique $N$. If there are multiple candidates, they have to be compared using (2).

Figure 4 depicts a case with linear preferences where there is indeterminancy between two levels of $N$ so the decentralized solution may give under provision of one organization or the optimal outcome. Although the results from above still apply to many cost structures, it is now possible to create other solutions with appropriate choices of the $C$ function. We see from the Figure, however, that as long as costs are weakly increasing in organization size, we can never have a split up stable outcome with a number of organizations below the optimum. 
Figure 4: Social optimum and split up stable equilibrium with variable costs

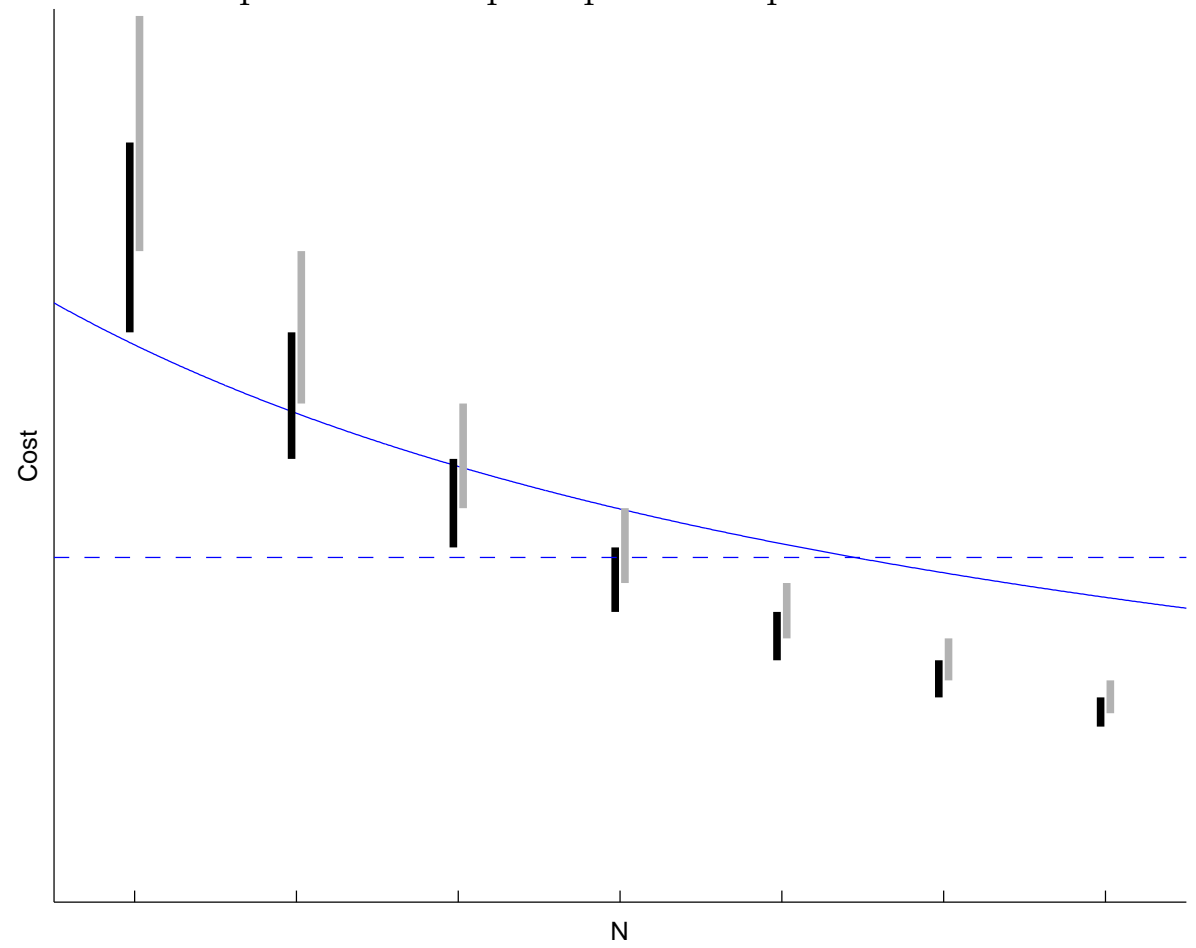

Notes: The black bars give intervals where $N$ organizations are socially optimal and the gray bars give intervals where $N$ is the smallest split up stable number of organizations. The solid line depicts a cost function that is linear in number of members whereas the dashed line represents pure fixed costs. 


\section{Equilibrium concepts}

As the split up stability concept is novel, it deserves some discussion. One way to think about this concept is a society that starts with one large organization, which subsequently splits until only minorities within each organization want further divisions. When this equilibrium is reach, we obtain the split up stable structure. This implies that split up stability is a suitable criterion when modeling organizational structures that can reasonably be thought of as emerging from one or a few mother organizations. This covers most types of associations, labor unions, political parties, and religious congregations. Fields where the number of organizations has grown over time, say because of increasing population or income, may plausibly be modeled using split up stability. For organizations emerging from the unification of many smaller units, such as the formation of countries, ${ }^{12}$ split up stability may be a less suitable criterion. This also applies to spheres where the number of organizations has been shrinking over time.

\subsection{Top down versus bottom up}

We could envisage the reverse of an organization splitting process, starting with a large number of organizations (or individual service production) that merge until an equilibrium is reached. Then mergers stop before the social optimum is reached, typically yielding too little merger and too many organizations. To see this, notice that a majority for two organizations to merge requires that the members at the center of each organization prefer a merger, which occurs when $u(0)-N C \leq u\left(\frac{1}{2 N}\right)-\frac{N C}{2}$. This gives a new set of critical cost levels

$$
M_{N}=\frac{2}{N}\left(u(0)-u\left(\frac{1}{2 N}\right)\right)
$$

where organizations merge until there is a number of organizations $N$ such that $M_{N+1}<$ $C \leq M_{N}$. It is trivial to see that $M_{N}>D_{N}$, so all equilibria that are reached in this way are split up stable. Also, as $\int_{0}^{\frac{1}{2 N}} u(x) d x \leq \frac{1}{N} u(0)$ and $(N+1) \int_{\frac{1}{2 N+2}}^{\frac{1}{2 N}} \geq \frac{1}{N} u\left(\frac{1}{2 N}\right)$, we have $M_{N}>S_{N}$, so the smallest merge stable number of organizations is larger than the socially optimal number of organizations. Graphically, this can be seen from Figure 2 where $M_{N}$ corresponds to a rectangle containing the whole shaded area. This equilibrium condition resembles Alesina and Spolaore's (1997) definition of B-equilibrium, altough they allow for voting on increasing the total number of organizations by one, and hence let the members internalize the subsequent change in membership structure. This is considered in more detail below.

When studying the number of countries, it seems reasonable to initially have a large number of extended households, who turn into villages, then chiefdoms, and through a long merging process into countries. Here, this merger equilibrium is a relevant equilibrium concept. For the types of organizations we study here, however, it seems more reasonable that we initially have one or a few organizations who gradually split up to suit the needs of the differentiated mass of members. Now split up stability is a more appropriate equilibrium concept.

\footnotetext{
${ }^{12}$ To study the process of countries splitting up, which is more common in the contemporary world, split up stability is a suitable tool.
} 


\subsection{Local and global split up stability}

The split up stability concept introduced in Section 2.2 is a local concept where the decision on whether to split the organization is taken without regard to the behavior of members of other organizations. However, if one organization chooses to split, this is going to have global effects: First the two new organizations attract new members from the adjacent organizations. As these organizations lose members of certain types, they are also going to change the type of good they provides which in turn leads to them attracting members from their adjacent organizations. Eventually, we end up with a configuration with equally sized organizations.

Fully rational agents might take this into account when forming preferences for splitting the organization. When a secessionist group make their calculations, they do not expect to stay smaller than the other groups in the future, but manage to fully anticipate the new organizational structure. Notice that this also implies that all organizational locations (centers) are changed by the secession. I refer to an equilibrium where effects on the whole organizational structure is taken into account as global split up stability.

The costs structure required to avoid a secession in this case is different from those considered above. Below, I show that in general costs need to be higher to avoid secession under the new global version of split up stability. I focus on the case of weakly concave preferences as convex preferences imply an inherent instability within the organization, cf. Section 2.2.2.

Consider the case of $N$ organization. The for any $i \in\{0, \ldots, N-1\}$, the organization attracting members from the interval $\left[\frac{i}{N}, \frac{i+1}{N}\right]$ is of type $\frac{i+\frac{1}{2}}{N}$. If this organization is split and society ends up with $N+1$ organizations, the members of the initial organization is split between the new organizations covering the intervals $\left[\frac{i}{N+1}, \frac{i+1}{N+1}\right]$ and $\left[\frac{i+1}{N+1}, \frac{i+2}{N+1}\right]$. As above, weakly concave preferences imply that members close to the center of the initial organization are the least in favor of splitting the organization.

However, preferences for splitting are no longer necessarily proportional to the distance from the initial organization type as the new organizational structure is different from the original one. The exception is the case with an odd $N$ and the middle organization covering the interval $\left[\frac{N-1}{2 N}, \frac{N+1}{2 N}\right]$, which still maintains symmetry and may serve as a benchmark for attitudes to splitting.

It follows that not all organizations are equally prone to splitting up. Specifically, organizations close to the center $\frac{1}{2}$ are the most prone to splitting. The intuition behind this result is that in case of a split, a large part of the fringe of both of the new organizations, where members are the least satisfied with the new structure, is located inside other organization. In one way, by splitting, the organization is capable of securing the best part of the new organizations for themselves. If an organization located close to 0 or 1 is split, most members remain in the same organization with the exception of a small group that ends up in the fringe of a new organization, obtaining at best minor gains.

To see this formally, compare the middle organization to one with center slightly below $\frac{1}{2}$. In the event of a split, the members of the middle organization are split between organizations located at $q_{1}=\frac{1}{2}-\frac{1}{2(N+1)}$ and $q_{2}=\frac{1}{2}+\frac{1}{2(N+1)}$. Define $x_{1}$ and $x_{2}$ with $x_{1}<\frac{1}{2}<x_{2}$ so that members located at these points are indifferent between splitting 
and not splitting. Then we have

$$
\begin{aligned}
& u\left(x_{1}-q_{1}\right)-C=u\left(\frac{1}{2}-x_{1}\right) \\
& u\left(q_{2}-x_{2}\right)-C=u\left(x_{2}-\frac{1}{2}\right)
\end{aligned}
$$

Compare this initial organization with one whose center is below $\frac{1}{2}$, which corresponds to the case where both $q_{1}$ and $q_{2}$ are increased by the same quantity $d q$. We get $\frac{d x_{1}}{d q_{1}}=\frac{d x_{2}}{d q_{2}}$, but as $\frac{d^{2} x_{1}}{d q_{1}^{2}} \leq 0 \leq \frac{d^{2} x_{2}}{d x_{2}^{2}}$, the subset of the organization opposing a split $x_{2}-x_{1}$ grows as $d q$ grows. Hence all organizations with center above $\frac{1}{2}$ have less support for splitting than the one at $\frac{1}{2}$. A similar reasoning holds for all organizations with types below $\frac{1}{2}$. Consequently, to study split up stability, we can focus on stability in the organization located at $\frac{1}{2}$ in the case of $N$ being odd and the organizations located at $\frac{N-1}{2 N}$ and $\frac{N+1}{2 N}$ in the case if $N$ being even.

Split up stability now requires that within each organization, there is only a minority that advocates a split. This yields a sequence of critical values $D_{N}^{G}$ such that if $C<D_{N}^{G}$, $N$ organizations is stable in the sense that there is not a majority in any organization for a split. As we saw above, members of organizations close to $\frac{1}{2}$ have more to gain from a split, so in reality split up stability is determined by whether there is a majority in the organization located at $\frac{1}{2}$ (in the case of $N$ even) or the two located at $\frac{1}{2} \pm \frac{1}{2 N}$ (in the case of $N$ odd).

A key question is whether this global perspective has an impact on the total number of organization. As the result below summarizes, it does indeed:

Proposition 4. When preferences are weakly concave, the critical cost values are higher with a global perspective, i.e. for any $N$ we have $D_{N}^{G} \geq D_{N}$.

Proof. To show that the proposition holds for all $N$, we need to consider the cases where $N$ is odd and even separately.

Case 1: $N$ is odd

If we assume an odd $N$, it suffices to verify that there is no majority for a split in the organization located at $\frac{1}{2}$. This holds whenever the members located at $\frac{1}{2} \pm \frac{1}{4 N}$ prefer no split. Hence whenever

$$
u\left[\left(\frac{1}{2}-\frac{1}{4 N}\right)-\left(\frac{1}{2}-\frac{1}{2(N+1)}\right)\right]-C \leq u\left[\frac{1}{2}-\left(\frac{1}{2}-\frac{1}{4 N}\right)\right]
$$

holds, the organization is split up stable. This gives the sequence of critical values

$$
D_{N}^{G}=u\left(\frac{N-1}{2 N(N+1)}\right)-u\left(\frac{1}{4 N}\right)
$$

In the case of $N=1$, it follows trivially that $D_{1}^{G}=D_{1}$. For $N \geq 3$, notice first that $D_{N}^{G}>D_{N}$ whenever

$$
u\left(\frac{N-1}{2 N(N+1)}\right) \geq \frac{u(0)+(N-1) u\left(\frac{1}{4 N}\right)}{N}
$$


By the weak concavity of $u$, we have

$$
u\left(\frac{N-1}{2 N(N+1)}\right) \geq \frac{N-1}{N+1} u(0)+\frac{2}{N+1} u\left(\frac{1}{4 N}\right)
$$

As $u(0)>u\left(\frac{1}{4 N}\right)$ and $\frac{N-1}{N+1}>\frac{1}{N}$, the expression always hold.

Case 2: $N$ is even

Now the pressure for splitting is strongest in the organizations with locations $\frac{1}{2}-\frac{1}{2 N}$ and $\frac{1}{2}+\frac{1}{2 N}$. Consider without loss of generality the first. In case of a split, new organizations are formed with centers located at $\frac{1}{2}-\frac{1}{2(N+1)}$ and $\frac{1}{2}$. There are pivotal members located at $x_{1}$ and $x_{2}$ defined by

$$
\left\{\begin{array}{r}
u\left(\left|\frac{1}{2}-\frac{1}{2(N+1)}-x_{1}\right|\right)-C=u\left(\frac{1}{2}-\frac{1}{2 N}-x_{1}\right) \\
u\left(\frac{1}{2}-x_{2}\right)-C=u\left(x_{2}-\frac{1}{4}-\frac{1}{2 N}\right)
\end{array}\right.
$$

We first need to show that $x_{1} \geq \frac{1}{2}-\frac{1}{2(N+1)}$. To see this, assume first that $x_{1}=$ $\frac{1}{2}-\frac{1}{2(N+1)}$ so $C=u(0)-u\left(\frac{1}{2 N(N+1)}\right)$ and $x_{2}=x_{1}+\frac{1}{2 N}=\frac{1}{2}-\frac{1}{2 N(N+1)}$. However, the agent at $x_{2}$ prefers a split if $u\left(\frac{1}{2 N(N+1)}\right)-u\left(\frac{2}{2 N(N+1)}\right) \geq C$. By the concavity of $u$, $u\left(\frac{1}{2 N(N+1)}\right)-u\left(\frac{2}{2 N(N+1)}\right) \geq u(0)-u\left(\frac{1}{2 N(N+1)}\right)$ this always hold. If $u$ is linear, the expression holds with equality in which case $x_{1}=\frac{1}{2}-\frac{1}{N+1}$. With a strictly concave $u$ the inequalities are strict, in which case it follows that $x_{1}>\frac{1}{2}-\frac{1}{N+1}$.

Second, inserting $x_{2}=\frac{1}{2 N}+x_{1}$ in the second equation of the system above, we get $u\left(\frac{1}{2}-\frac{1}{2 N}-x_{1}\right)-C=u\left(x_{1}\right)$. Added to the first equation, this yields $2 C=$ $u\left(x_{1}-\frac{1}{2}-\frac{1}{2(N+1)}\right)-u\left(x_{1}\right)$. By the concavity of $u$, this expression is increasing in $x_{1}$ and hence minimized at $x_{1}=\frac{1}{N+1}$. Hence we get $D_{2}^{G} \geq \frac{1}{2}\left[u(0)-u\left(\frac{1}{N+1}\right)\right]$. As for all $N \geq 2$ we have $\left[u(0)-u\left(\frac{1}{N+1}\right)\right] \geq\left[u(0)-u\left(\frac{1}{2 N}\right)\right]$ and $\frac{1}{2} \frac{1}{N}$, it follows that $D_{2}^{G} \geq D_{2}^{G}$.

The intuition for the result is that under the global view, the increase in costs from a split is lower as the perceived decrease in organization size is smaller. This is partially offset by smaller reduction in travel costs, but this is not enough to overturn the effect.

The Proposition implies that there are stricter requirements for $N$ organizations being stable when members have a global view than the local view considered above. It follows that there are values of $C$ so a larger number of organizations is required for stability with a global view.

In the case of linear preferences, we can quantify the over provision of organizations. In this case, preferences are given by $u(x)=1-a x$. We first notice that the pressure for splitting is the same across organizations. In the organization covering $\left[\frac{i}{N}, \frac{i+1}{N}\right]$, members with types $x<\left(i+\frac{1}{2}\right) \frac{2 N+1}{N(N+1)}-\frac{C}{a}$ and $x>\left(i+\frac{1}{2}\right) \frac{2 N+1}{N(N+1)}+\frac{1}{N+1}+\frac{C}{a}$ prefer to split the organizations and the remaining members prefer to keep the initial configuration. There is a majority for splitting if the mass of members preferring a split is above $\frac{1}{2 N}$, which yield $D_{N}^{G}=\frac{a}{2 N(N+1)}$. Let $\lceil\cdot\rceil$ denote the ceiling function (i.e. the smallest following integer). Then for some cost $C$ the smallest number of organizations that is split up stable under the local definition is $N_{C}=\left\lceil\sqrt{\frac{a}{4 C}}\right\rceil$ whereas under the global definition we get $N_{C}^{G}=\left\lceil\sqrt{\frac{a}{2 C}+\frac{1}{4}}-\frac{1}{2}\right\rceil$. At $\frac{a}{C}=4$ both definitions yield $N=1$. But if we let costs 
decline, the number of definitions required for global split up stability grows faster than the number required for local split up stability. In the limit, we get $\sqrt{2}$ times the optimal number of organizations, as $\frac{a}{C} \rightarrow+\infty$ so $\frac{N_{C}^{G}}{N_{C}} \rightarrow \sqrt{2} .^{13}$ This is close to the results found in the model of Alesina and Spolaore (1997).

\subsection{The core and threats of secessions}

One could object that split up stability's requirement of a majority vote to split is conservative. An alternative approach is to see whether there are possibilities of secessions. This essentially corresponds to studying whether the equilibrium is in the core (cf. Paúly's (1967) approach to stability in club economies). In the economy we study, the core consists of numbers of organization where no subgroup can secede and form a new organization and where no two groups can merge and improve the welfare of all the members.

Consider first other threats of unilateral secessions: If there is no majority to split the organization in 2 organizations, there is no majority to split it into $q>2$ organizations. One can also easily verify that with weakly concave preferences, no single group would unilaterally want to quit the organization if (4) holds. The reason is that the member at $\frac{1}{2 N}$ is the most reluctant to a split, so he and all other members in $\left[\frac{1}{4 N}, \frac{3}{4 N}\right]$ do not support any split up. The new organization would hence get a size below $\frac{1}{2 N}$, which would give it larger costs than the majority vote of splitting in two. Hence no such group could be formed.

Split up stable equilibria are also robust against a coalition of members from two adjacent organizations in most cases. Consider a potential new organization located at a point where the member is indifferent between two organizations where it can attract members from both organizations. We should ask wether an individual located at a distance $x$ is willing to join the new organization when members at distances in $[0, x)$ remain in the previous organization whereas members in $\left(x, \frac{1}{2 N}\right]$ join the new organization. If this is the case for some $x$, formation of a new organization is possible. The utility of joining the new organization is $u\left(\frac{1}{2 N}-x\right)-\frac{C}{2\left(\frac{1}{2 N}-x\right)}$, so an individual joins the new organization whenever

$$
\left[u\left(\frac{1}{2 N}-x\right)-u(x)\right] \frac{\frac{1}{2 N}-x}{N x}>C
$$

As the initial equilibrium is split up stable, we also know that

$$
C>\frac{u(0)-u\left(\frac{1}{4 N}\right)}{N}
$$

so a new organization is viable if there is some $x$ such that

$$
\frac{u\left(\frac{1}{2 N}-x\right)-u(x)}{u(0)-u\left(\frac{1}{4 N}\right)}>\frac{x}{\frac{1}{2 N}-x}
$$

Proposition 5. In split up stable equilibria with with equally sized organizations and linear or convex preferences, formation of new organizations at the boundary between organizations is not viable.

\footnotetext{
${ }^{13}$ As the socially optimal number of organizations is given by $\left\lceil\sqrt{\frac{a}{4 C}+\frac{1}{4}}-\frac{1}{2}\right\rceil$ a similar result holds when comparing to the the optimal outcome.
} 
Proof. Consider first the case of linear preferences $u(x)=1-a x$. Then the total membership in the new organization is $2\left(\frac{1}{2 N}-x\right)$ so the member located at $x$ derives utility

$$
1-a\left(\frac{1}{2 N}-x\right)-\frac{C}{2\left(\frac{1}{2 N}-x\right)}
$$

which is to be compared with the utility level $1-a x-C N$ obtained in the old organization. It is optimal to join the secessionists whenever $C<\frac{a}{N}\left(2 x-\frac{1}{2 N}\right)\left(\frac{1}{2 N}-x\right) \leq \frac{1}{32 N^{2}}$. However, when $N$ organizations is the largest split up stable number of organizations, we have $C>\frac{a}{4(N-1)^{2}}$. As $\frac{a}{4(N-1)^{2}}>\frac{1}{32 N^{2}}$ for all $N \in \mathbb{N}$, the condition is never satisfied.

If the utility function $u$ is convex, we compare it to the linear counterpart $\tilde{u}$ with $\tilde{u}(0)=u(0)$ and $\tilde{u}\left(\frac{1}{2 N}\right)=u\left(\frac{1}{2 N}\right)$. Then by convexity $u(0)-u(z)>\tilde{u}(0)-\tilde{u}(z)$ and $u(z)-u\left(\frac{1}{2 N}\right)<\tilde{u}(z)-\tilde{u}\left(\frac{1}{2 N}\right)$ for all $z$. Hence the numerator of the LHS of (11) decreases and the numerator increases with convexity and the condition still holds.

Bogomolnaia et al. (2007) show that with heterogeneous organization sizes, the formation of new organizations may get support from groups within the old organizations but without support at the border. In such cases the Proposition may not go through, but are ruled out in the curren setting.

As the proof for the linear case holds with a margin, the Proposition also applies to a class of concave preferences that are only weakly concave. It is not difficult, however, to find sufficiently concave preferences that there is a possibility of successful secessions. The main reason for this is that the median voters within the organizations, located at $\frac{1}{4 N}$ and $\frac{3}{4 N}$, are less hit by their distance to the middle of the organization than the members further away, and hence less enthusiastic to splitting the organization. This is also the case where split up stability may lead to underprovision of organizations.

The concept of split up stability may also be seen as a variety of "secession proofness" considered by Le Breton and Weber (2003) and Le Breton et al. (2005). ${ }^{14}$ Secession proofness requires that there is no coalition of member $S$ within one organization such that all members in $S$ are better off if $S$ were to become a separate organization. This is somewhat weaker than split up stability. Secession proof equilibria may exhibit efficiency properties similar to those of split up stability under the assumptions studied by Le Breton and Weber (2003) and Le Breton et al. (2005). However, under the conditions studied in the present paper, there may be cases of secession proof arrangements that still display too few organizations compared to the social optimum. Specifically, there are cost levels such that no single group find it profitable to break out, but where there is a majority for a split. To obtain a majority to split an organization, the members favoring the split do not have to break out into the same organization as they would under the secession proofness test. Hence the requirement is weaker and allows for a larger number of organizations.

Related to the core is also the competitive clubs approach of Ellickson et al. (1999, 2001, 2006). As in my model, they consider a continuum of heterogeneous agents. The main difference is that whereas I have a finite number of organizations with a continuum of members, they have a continuum (or a large number, in the case of Ellickson et al.

\footnotetext{
${ }^{14}$ When organization membership is allowed to vary between organizations, Drèze et al. (2007) show that only a "Rawlsian" arrangement where the sum of transport costs and membership fees are equal for all is secession proof. Drèze et al. (2008) extend the analysis of secession proofness to a multidimensional setting and show the impossibility of constructing an arrangement that is secession proof without external transfers.
} 
(2001)) of organizations each with a finite number of members. In this way, they can legitimate a market solution and find membership fees in general equilibrium. As there is no crowding in my model, multiple organizations producing the same service would be pure waste and never occur in equilibrium. As the competitive approach gives an infinite number of small organizations, a direct comparison of outcomes is not possible.

\section{Voluntary membership}

\subsection{Social optimum}

The model where everybody belongs to an organization is unrealistic unless the service provided is extremely valuable or there are legal obligations to belong to an organization. To endogenize the membership decisions, we need to introduce an outside option to capture the value of not joining any organization, for instance by abstaining from consuming the good provided by the organization or providing a substitute privately. In this part of the analysis I restrict attention to linear preference structures primarily because this largely simplifies the analysis. Moreover, the issues of non-linear preferences and endogeneous membership are somewhat orthogonal so the gains from analyzing them together are probably limited and is left for future research.

To study voluntary membership, we extend the types of agents to a two-dimensional space $(x, \varepsilon)$ where as before $x$ is the organizational type with a uniform distribution on $[0,1]$ and $\varepsilon$ is a variable, assumed to be uniformly distributed on the unit interval, representing the utility of not belonging to an organization. An individual will join an organization if, for the optimal choice of organization $i, U[x, q(x)]-c>\varepsilon$ where $c$ is the membership fee (which is identical in all organizations). Hence the fraction of agents of type $x$ who belong to an organization is $U[x, q(x)]-c$ whenever this falls in the unit interval.

As before, the social planner's problem is to choose the number of organizations and their types to maximize

$$
\max _{N,\left\{q_{i}\right\}} \int_{0}^{1} \int_{0}^{1} \max [u(|x-q(x)|), \varepsilon]-N C d \varepsilon d x .
$$

It is seen that in this problem, the optimal types of organizations for given $N$ is still given by Proposition 1. As preferences are linear, i.e. $u(x)=1-a x$ with $0<a \leq 1$, the sum of benefits for all individuals when there are $N$ organizations is

$$
\begin{aligned}
W(N) & =2 N \int_{x=0}^{1 / 2 N} \int_{u=0}^{1} \max (1-a x, u) d u d x \\
& =1-\frac{a}{4 N}+\frac{a^{2}}{24 N^{2}} .
\end{aligned}
$$

The optimal number of organizations is the integer $N$ that maximizes $W(N)-N C$. The cut off cost levels $S_{N}$, so $N$ organizations is optimal when $C \in\left[S_{N}, S_{N-1}\right)$, are found by solving $W(N)=W(N+1)$. This yields

$$
S_{N}=\frac{a}{4 N(N+1)}-\frac{a^{2}(2 N+1)}{24 N^{2}(N+1)^{2}} .
$$

In $(a, C)$-space the sets $\left[S_{N}, S_{N-1}\right]$ are the areas between parabolae. Comparing it to the 
$S_{N}$ we found for exogenous membership in equation (3), it is seen that the we require a lower cost to obtain the same number of organizations when membership is endogeneous. This means that the optimal number of organizations is (weakly) lower under endogeneous membership.

\subsection{Membership in the decentralized solution}

Before we can discuss split up stability in the decentralized solution, we need to study the pattern of membership for a given number $N$ of organizations. Without loss of generality, consider the organization at $\left[0, \frac{1}{N}\right]$, where the good produced is of type $\frac{1}{2 N}$. As membership is now endogeneous and members with types close to the center of the organization derive more utility from joining than members closer to the boundaries, a larger fraction of agents with types close to $\frac{1}{2 N}$ are members. Particularly, the fraction of agents of type $x$ who are members is implicitly defined by

$$
\psi_{N}(x)=\max \left\{1-a\left|x-\frac{1}{2 N}\right|-\frac{C}{\int_{0}^{1 / N} \psi_{N}(x) d x}, 0\right\} .
$$

As $\psi_{N}$ is symmetric around $\frac{1}{2 N}$ on $\left[0, \frac{1}{N}\right]$, the median member of the organization is located at $\frac{1}{2 N}$. The median voter theorem still applies, so the variety chosen by the organization is the variety in the center, $\frac{1}{2 N}$. To study membership in equilibrium, notice that total membership in an organization $\Psi_{N}=\int_{0}^{1 / N} \psi_{N}(x) d x$, solves

$$
\Psi_{N}=2 \int_{0}^{1 / 2 N} \max \left\{1-a x-\frac{C}{\Psi_{N}}, 0\right\} d x .
$$

If $\Psi_{N}$ exists, the existence of $\psi_{N}$ follows trivially. The fraction $\Psi_{N}$ is a fixed point of the mapping

$$
\Lambda: \Psi_{N} \mapsto 2 \int_{0}^{1 / 2 N} \max \left\{1-a x-\frac{C}{\Psi_{N}}, 0\right\} d x .
$$

This mapping can be rewritten

$$
\Lambda(\Psi)= \begin{cases}0 & \text { if } \Psi<C \\ \frac{1}{a}\left(1-\frac{C}{\Psi}\right)^{2} & \text { if } C \leq \Psi<\frac{C}{1-\frac{a}{2 N}} \\ \frac{1}{N}\left(1-\frac{a}{4 N}-\frac{C}{\Psi}\right) & \text { if } \frac{C}{1-\frac{a}{2 N}} \leq \Psi\end{cases}
$$

The proof is provided in Appendix A.2.

The mapping $\Lambda$ is strictly increasing on $[C, 1]$, continuous, and continuously differentiable for all $\Psi_{N} \neq C$. Equation (14) has a trivial solution at $\Psi_{N}=0$, and for some parameter configurations there is also another equilibrium.

Proposition 6. If the equation $N \Psi_{N}^{2}-\left(1-\frac{a}{4 N}\right) \Psi_{N}+C=0$ has real roots, the equation $\Psi_{N}=\Lambda\left(\Psi_{N}\right)$ has a unique stable interior equilibrium given by

$$
\Psi_{N}^{*}=\frac{\left(1-\frac{a}{4 N}\right)+\sqrt{\left(1-\frac{a}{4 N}\right)^{2}-4 N C}}{2 N}
$$

The proof consists in showing that we always have $\frac{C}{1-\frac{a}{2 N}} \leq \Psi$, and hence that $\Psi^{*}$ 
solves a second order equation. The details are provided in Appendix A.3.

\subsection{The split up stable outcome}

The concept of split up stability is still applicable and useful, but a new complication arises when considering endogeneous membership. Consider again without loss of generality the first organization covering $\left[0, \frac{1}{N}\right]$. There are no longer equally many members of each type as $\psi$ has a peak at $\frac{1}{2 N}$. The majority for a split up decision is now not determined by the members of types $\frac{1}{4 N}$ and $\frac{3}{4 N}$, but a set of voters closer to the center of the organization. This tends to reduce the pressure for splitting the organization, and also reduce the equilibrium number of organizations. In addition to this effect, the fact that fewer agents join organization because they have outside opportunities pull in the same direction. However, as organizations only consist of agents with relatively bad outside opportunities, they tend to over value organizations. This effect pull in the direction of too many organizations. I first show that the latter two effects almost cancel out. I then go on to studying the first effect in Section 4.4. Hence for the time being, I condition on pivotal voters of types $\frac{1}{4 N}$ and $\frac{3}{4 N}$. These members prefer not splitting when ${ }^{15}$

$$
\frac{a}{4 N}+\frac{C}{\Psi_{N}} \leq \frac{C}{\Psi_{2 N}}
$$

From this expression, we can find the smallest split up stable number of organization for any set of parameters:

Proposition 7. With fixed pivotal voters, $N$ organizations is split up stable when

$$
C \geq D_{N}:=\frac{8 N a-15 a^{2}+3 a \sqrt{64 N^{2}-48 N a+25 a^{2}}}{128 N^{3}}
$$

The proof, which is based on squaring (16) twice and simplifying, is provided in Appendix A.4.

As in Section 2.2.1, this provides a partitioning of $(a, C)$-space where $N$ is the smallest split up stable number of organizations when $C \in\left[D_{N}, D_{N-1}\right)$. Comparing this interval to $\left(S_{N}, S_{N-1}\right)$, we are ready to analyze the provision of organizations in the decentralized case. The main finding is:

Proposition 8. With fixed pivotal members, for any number of organizations $N$, the limiting cost for split up stability $D_{N} \in\left(S_{N}, S_{N-1}\right)$ so an equilibrium with the social optimal $N$ or over provision of 1 organization is split up stable.

The proof, which is mainly algebraic simplifications of a comparison of equations (13) and (16), is provided in Appendix A.5.

The result is illustrated in Figure 5. The proposition shows that the smallest split up stable equilibrium either corresponds to the social optimum or under provision of one organization, as was the case with full membership. There are two opposing forces altering the outcome relative to exogeneous membership: First, members of organizations tend to have relatively bad outside opportunities and value the services of the organizations above

\footnotetext{
${ }^{15}$ A sustainable number of organizations $N$ has to satisfy $\Psi_{N}=\Lambda\left(\Psi_{N}\right)$. One could imagine this to be a stricter criterion than equation (16). But using the ensuing number of organizations, it is easily verified that this is not the case.
} 
Figure 5: Combinations of $a$ and $C$ that yield $N$ organizations with voluntary organization membership.

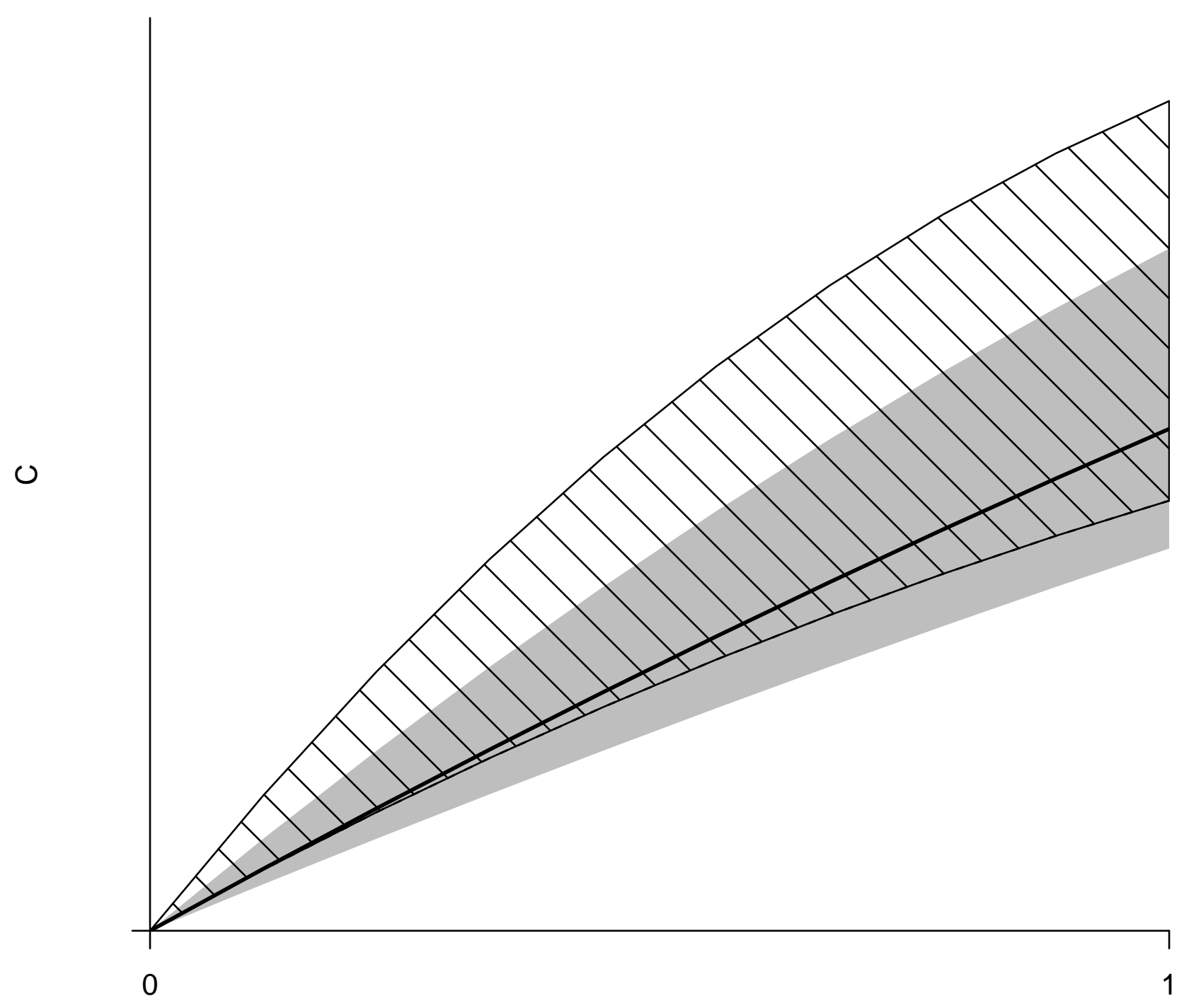

a

Notes: The gray area shows the area $\left[S_{N}, S_{N-1}\right)$ where $N$ organizations are socially optimal, and the hatched area is the set $\left[D_{N}, D_{N-1}\right)$ where $N$ is the smallest split up stable number of organizations. Ignoring the integer constraint, exactly $N$ organizations is optimal along the solid line. 
average. The social planner acknowledges that there are outside opportunities, and, as seen above, reduces the number of organizations relative to the number with exogeneous membership. This is not acknowledged by the actual members of the organizations, and tend to give too many organizations. Second, as some choose to not join organizations, so $\Psi_{N}<1 / N$, there is an increased cost per member for a given number of organizations, tending to give too few organizations. The two effects almost cancel out, but the latter is somewhat stronger.

\subsection{The pivotal members}

The analysis so far has been conditional on the members pivotal in the split up decision being fixed at the positions they had with exogeneous membership. However, as membership varies with each individual's evaluation of the organization's appropriateness, a larger share of individuals are members close to the organization center. The position of the pivotal members are given by the following lemma:

Lemma 1. The pivotal members are of types $\frac{1}{2 N} \pm m$ where

$$
m=\frac{\left(1-\frac{C}{\Psi_{N}}\right)-\sqrt{\left(1-\frac{C}{\Psi_{N}}\right)^{2}-\frac{a}{2 N}\left(1-\frac{a}{4 N}-\frac{C}{\Psi_{N}}\right)}}{a}
$$

Proof. As $\psi$ is symmetric around $\frac{1}{2 N}$ on $\left[0, \frac{1}{N}\right]$, there is some $m$ such that the pivotal members are of types $\frac{1}{2 N} \pm m$. As we need an equal mass of members on $[0, m]$ as on $\left[m, \frac{1}{2 N}\right]$, we have

$$
\int_{0}^{m} \max \left\{1-a x-\frac{C}{\Psi_{N}}, 0\right\} d x=\int_{m}^{1 / 2 N} \max \left\{1-a x-\frac{C}{\Psi_{N}}, 0\right\} d x .
$$

From Proposition $7, \psi(x)>0$ for all $x$, so the expression reduces to $\int_{0}^{m} 1-a x-\frac{C}{\Psi_{N}} d x=$ $\int_{m}^{1 / 2 N} 1-a x-\frac{C}{\Psi_{N}} d x$. Integrating and solving, this reduces to the quadratic equation

$$
a m^{2}-2 m\left(1-\frac{C}{\Psi_{N}}\right)+\frac{1}{2 N}\left(1-\frac{C}{\Psi_{N}}-\frac{a}{4 N}\right)=0 .
$$

Only the smaller root guarantees $m \in\left[0, \frac{1}{4 N}\right]$.

If the organization decides to split, it is as before split in two equal organizations, yielding new organization centra at $\frac{1}{4 N}$ and $\frac{3}{4 N}$. Hence the pivotal members prefer no split whenever

$$
a m+\frac{C}{\Psi_{N}} \leq a\left(\frac{1}{4 N}-m\right)+\frac{C}{\Psi_{2 N}}
$$

This expression no longer permits a closed form solutions for the cut off cost. However, we can show that the conclusions from Proposition 8 still holds:

Proposition 9. With pivotal members determined as in Lemma 1, we have a limiting cost for split up stability $D_{N} \in\left(S_{N}, S_{N-1}\right)$ so an equilibrium with the social optimal $N$ or over provision of 1 organization is split up stable. 
Proof. It is trivial from comparing (16) with (19) that the lowest cost for split up stability decreases so $D_{N}<S_{N-1}$ follows from Proposition 8. The proof of $D_{N}>S_{N}$ is provided in Appendix A.6.

In addition to the two changes from the exogneous case mentioned in Section 4.3, there is now a third factor, the change in the identity of the pivotal voters, which also tend to limit the number of organizations. This effect is not strong enough to change the above conclusions. However, as this reduces the demand for organizations, it increases the likelihood that the exact number of organizations is formed (in the sense that the critical level of $C$ is reduced). But we may still get over provision of one organization.

\section{Concluding remarks}

We have studied organization formation in heterogeneous societies. To define an equilibrium number of organizations, we say that organizations split until the stage where only a minority within each organization prefers further divisions. This condition is referred to as split up stability. One main result of the paper is that with linear preferences, the socially optimal number of organizations is either achieved fully, or there is over provision of a single organization. In an extension with a heterogeneous outside option, I show that this result also hold with voluntary membership in organizations. If instead preferences are concave, so organizations cater to a broad set of individuals, we may get under provision of organizations. In the opposite case where preferences are convex and organizations specialized, both over and under provision may occur.

When the number of organizations is large and preferences close to linear, the relative mis-allocating is small and there is little need for public interventions. However, taxation or subsidies of organizations may be used to achieve the exact social optimum. The need for this is largest in cases with few organizations where the relative over provision may be large and when organizations are either very specialized (convex preferences) or very broad (concave preferences).

It is not obvious that these optimality properties would remain in a generalized version of the model. More general cost structures seem to have little effect on the properties of the decentralized solution. The distribution of the outside opportunities, however, may potentially have major impacts. With non-uniform distributions, closed form solutions are generally not available, so general conclusions are difficult to draw. Another extension would be to allow for non-uniformly distributed agents or multi-dimensional heterogeneity. Existence and stability become more complicated, but this is of course a more precise depiction of real societies. This could also create an opportunity for multiple layers of organizations.

\section{A. Proofs}

\section{A.1. A result for convex preferences}

The proof that the agents located at $\frac{1}{4 N}$ and $\frac{3}{4 N}$ are more wiling to split the organization with convex preferences than with linear utility is the converse to the proof to Proposition 3:

We compare preferences given by $u$ to a linear version given by $\tilde{u}(x)=u(0)-$ $2 N\left[u(0)-u\left(\frac{1}{2 N}\right)\right] x$. Denote by $\tilde{D}_{N}$ the cut offs in the decentralized solution if the agents located at $\frac{1}{4 N}$ and $\frac{3}{4 N}$ could dictate the splitting outcome. 
The proof is in two steps; I first show that the proposition holds for a special class of utility functions, and then that it holds for all convex preferences if it holds for this class.

1) Consider the class of utility functions

$$
u_{\gamma}(x)= \begin{cases}u(0)-(2 N+2 \gamma)\left[u(0)-u\left(\frac{1}{2 N}\right)\right] x & \text { if } x<\frac{1}{2 N+2 \gamma} \\ u\left(\frac{1}{2 N}\right) & \text { if } x \geq \frac{1}{2 N+2 \gamma}\end{cases}
$$

for $\gamma \in[0,1]$. The corresponding socially optimal cut offs are

$$
S_{N}^{\gamma}=2 \int_{0}^{\frac{1}{2 N+2}} u_{\gamma}(x) d x-2 N \int_{\frac{1}{2 N+2}}^{\frac{1}{2 N}} u_{\gamma}(x) d x=\frac{N+2 \gamma-\gamma^{2}}{2(N+1)(N+\gamma)}\left[u(0)-u\left(\frac{1}{2 N}\right)\right]
$$

The function $u_{0}=\tilde{u}$, the linear utility function. The corresponding cut offs for split up stability are

$$
\tilde{D}_{N}^{\gamma}=\frac{u_{\gamma}(0)-u_{\gamma}\left(\frac{1}{4 N}\right)}{N}=\frac{N+\gamma}{2 N^{2}}\left[u(0)-u\left(\frac{1}{2 N}\right)\right]
$$

As already seen in Proposition 2, $S_{N}^{0}<D_{N}^{0}$, i.e. there may be over provision with linear preferences. We have

$$
\frac{\partial S_{N}^{\gamma}}{\partial \gamma}=\frac{N-2 \gamma N-\gamma^{2}}{2(N+1)(N+\gamma)^{2}}\left[u(0)-u\left(\frac{1}{2 N}\right)\right]
$$

and

$$
\frac{\partial \tilde{D}_{N}^{\gamma}}{\partial \gamma}=\frac{1}{2 N^{2}}\left[u(0)-u\left(\frac{1}{2 N}\right)\right]
$$

so for all $\gamma \in[0,1], \frac{\partial S_{N}^{\gamma}}{\partial \gamma}<\frac{\partial \tilde{D}_{N}^{\gamma}}{\partial \gamma}$ which proves the Proposition for preferences described by $u_{\gamma}$.

2) For any convex utility function $u$, take $\hat{\gamma}=(N+1) \frac{u(0)-u\left(\frac{1}{2 N+2}\right)}{u(0)-u\left(\frac{1}{2 N}\right)}+N$ so $u\left(\frac{1}{2 N+2}\right)=$ $u_{\hat{\gamma}}\left(\frac{1}{2 N+2}\right)$. On $\left[0, \frac{1}{2 N+2}\right] u_{\hat{\gamma}}$ forms an arc to $u$ so $u(x) \leq u_{\hat{\gamma}}(x)$ for all $x$ in this interval. Hence

$$
\int_{0}^{\frac{1}{2 N+2}} u(x) d x \leq \int_{0}^{\frac{1}{2 N+2}} u_{\hat{\gamma}}(x) d x
$$

Furthermore, for a small $\epsilon>0$ we also have $u\left(\frac{1}{2 N+2}-\epsilon\right) \leq u_{\hat{\gamma}}\left(\frac{1}{2 N+2}-\epsilon\right)$ so $u^{\prime}\left(\frac{1}{2 N+2}\right) \leq$ $u_{\hat{\gamma}}^{\prime}\left(\frac{1}{2 N+2}\right)$. For all $x \in\left[\frac{1}{2 N+2} \frac{1}{2 N+2 \gamma}\right]$ we have $u^{\prime \prime}(x) \leq 0$ by convexity and $u_{\hat{\gamma}}^{\prime \prime}(x)=0$ by assumption, so $u^{\prime \prime}(x) \leq 0>u_{\hat{\gamma}}^{\prime \prime}(x)=0$. Consequently, $u(x) \geq u_{\hat{\gamma}}(x)$ on this interval. As $u$ is decreasing, $u(x) \geq u\left(\frac{1}{2 N}\right)$ for all $x \in\left[\frac{1}{2 N+2 \gamma} \frac{1}{2 N}\right]$ whereas $u_{\hat{\gamma}}(x)=u\left(\frac{1}{2 N}\right)$ on this interval. Hence we have $u(x) \geq u_{\hat{\gamma}}(x)$ on this interval as well. It follows that

$$
\int_{\frac{1}{2 N+2}}^{\frac{1}{2 N}} u(x) d x \geq \int_{\frac{1}{2 N+2}}^{\frac{1}{2 N}} u_{\hat{\gamma}}(x) d x
$$


and hence that $S_{N} \leq S_{N}^{\hat{\gamma}}$.

Finally, as $u(x) \leq u_{\hat{\gamma}}(x)$ for all $x \in\left[0, \frac{1}{2 N+2}\right] u_{\hat{\gamma}}$, we have $u\left(\frac{1}{4 N}\right) \leq u_{\hat{\gamma}}\left(\frac{1}{4 N}\right)$ and hence $\tilde{D}_{N} \geq \tilde{D}_{N}^{\hat{\gamma}}$.

\section{A.2. Proof of equation (15)}

Consider first the case $\frac{a}{2 N}+\frac{C}{\Psi_{N}}<1$, so there are participants at all values of $x$. Then

$$
\begin{aligned}
\Psi_{N} & =2 \int_{0}^{1 / 2 N} 1-a x-\frac{C}{\Psi_{N}} d x \\
& =\frac{1}{N}-\frac{C}{N \Psi_{N}}-\frac{a}{4 N^{2}}
\end{aligned}
$$

When $\frac{a}{2 N}+\frac{C}{\Psi_{N}}>1$ and $\frac{C}{\Psi_{N}}<1$, there are some member types $x$ where no-one choose to join the organization and some member types $x$ where at least some agents join. We now get total member ship as

$$
\begin{aligned}
\Psi_{N} & =2 \int_{0}^{\frac{1}{a}\left(1-\frac{C}{\Psi_{n}}\right)} 1-a x-\frac{C}{\Psi_{N}} d x \\
& =\frac{1}{a}\left(1-\frac{C}{\Psi_{N}}\right)^{2}
\end{aligned}
$$

Finally, when $\frac{C}{\Psi_{N}}>1$, no-one wants to join so $\Psi_{N}=0$.

\section{A.3. Proof of Proposition 6}

The roots of $N \Psi_{N}^{2}-\left(1-\frac{a}{4 N}\right) \Psi_{N}+C=0$ are given by

$$
\Psi_{N}=\frac{\left(1-\frac{a}{4 N}\right) \pm \sqrt{\left(1-\frac{a}{4 N}\right)^{2}-4 N C}}{2 N}
$$

where only the larger root satisfies $\frac{C}{1-\frac{a}{2 N}} \leq \Psi_{N}$. We now need to show that (i) this root always satisfies $\frac{C}{1-\frac{a}{2 N}} \leq \Psi_{N}$, and (ii) that it is the unique interior solution of the equation.

To show (i), we know that $\left(1-\frac{a}{4 N}\right)^{2}>4 N C$ as the roots by assumption are real, so $C<\frac{1}{4 N}-\frac{a}{8 N}+\frac{a^{2}}{16 N^{2}}$. As $\Psi_{N} \leq \frac{1-\frac{a}{4 N}}{2 N}$, it suffices to show that $\frac{C}{1-\frac{a}{2 N}}<\frac{1-\frac{a}{4 N}}{2 N}$ which holds when $2 N C<\left(1-\frac{a}{4 N}\right)\left(1-\frac{a}{2 N}\right)$ i.e. when $C<\frac{1}{2 N}-\frac{3 a}{8 N^{2}}+\frac{a^{2}}{16 N^{2}}$. Then (i) holds when $\frac{1}{4 N}-\frac{a}{8 N}+\frac{a^{2}}{16 N^{2}}<\frac{1}{2 N}-\frac{3 a}{8 N^{2}}+\frac{a^{2}}{16 N^{2}}$, which holds when $0<\frac{1}{4 N}\left(1-\frac{a}{N}+\frac{3 a^{2}}{16 N^{2}}\right)$, which again holds when $a<\frac{4}{3}$. As $a \leq 1$ by assumption, we have $\frac{C}{1-\frac{a}{2 N}} \leq \Psi_{N}^{*}$.

To show (ii), it is easily seen that $\Lambda$ is convex for $\Psi_{N}<\frac{3 C}{2}$ and concave for $\Psi_{N}>\frac{3 C}{2}$. As $\Lambda$ is continuously differentiable at $\Psi_{N}=\frac{C}{1-\frac{a}{2 N}}$, concavity also hold in this point. The as $\Psi_{N}^{*}$ is real, (15) has two interior solutions, one stable and one unstable. 


\section{A.4. Proof of Proposition 7}

Whenever there are members of organization when there are $2 N$ organizations, the minimum sustainable number is determined by (16). Define

$$
\theta_{N}=\frac{1}{\Psi_{N}}=\frac{1-\frac{a}{4 N}-\sqrt{\left(1-\frac{a}{4 N}\right)^{2}-4 N C}}{2 C},
$$

so the split up stability condition becomes

$$
\begin{aligned}
\frac{a}{4 N} & =C \theta_{2 N}-C \theta_{N} \\
& =\frac{1}{2}\left[\left(1-\frac{a}{8 N}-\sqrt{\left(1-\frac{a}{8 N}\right)^{2}-8 N C}\right)-\left(1-\frac{a}{4 N}-\sqrt{\left(1-\frac{a}{4 N}\right)^{2}-4 N C}\right)\right]
\end{aligned}
$$

Taking squares of the expression and solving, we get

$$
\frac{192 C N^{3}-32 N^{2}+12 N a+a^{2}}{16 N^{2}}=-2 \sqrt{\left(\left(1-\frac{a}{4 N}\right)^{2}-4 N C\right)\left(\left(1-\frac{a}{8 N}\right)^{2}-8 N C\right)} .
$$

Taking squares again and solving yields the equation

$$
256 C^{2} N^{5}-32 C N^{3} a+60 C N^{2} a^{2}-8 N a^{2}+3 a^{3}=0 .
$$

This is a quadratic equation in $C$ where one the largest root gives a positive $C$, and hence yielding the solution (7).

To see that $2 N$ organizations is sustainable whenever this condition holds, notice that this holds iff $\left(1-\frac{a}{8 N}\right)^{2}-8 N C \geq 0$. Hence a sufficient condition is that $C^{D E} \leq \frac{a^{2}}{512 N^{3}}-$ $\frac{a}{32 N^{2}}+\frac{1}{8 N}$. Using the derived expression for the iso-organization line, this expression is satisfied when $12 a \sqrt{-48 N a+64 N^{2}+25 a^{2}} \leq 64 N^{2}-48 N a+61 a^{2}$. Taking squares and simplifying, this condition reduces to $0 \leq\left(64 N^{2}-48 N a-11 a^{2}\right)^{2}$ which is trivially satisfied for all real $a$ and $N$.

\section{A.5. Proof of Proposition 8}

Notice first that the socially optimal number of organizations disregarding the integer constraint yields a first order condition $S_{N}^{*}=\frac{a}{4 N^{2}}-\frac{a^{2}}{12 N^{3}}$ where of course $S_{N}<S_{N}^{*}<S_{N-1}$ We can show

$$
\frac{8 N a-15 a^{2} \pm 3 a \sqrt{-48 N a+64 N^{2}+25 a^{2}}}{128 N^{3}}<\frac{3 N a-a^{2}}{12 N^{3}}
$$

This holds whenever $24 N a-45 a^{2}+9 a \sqrt{-48 N a+64 N^{2}+25 a^{2}}<96 N a-32 a^{2}$ or $81\left(-48 N a+64 N^{2}+25 a^{2}\right)<(72 N+13 a)^{2}$. For $a>0$, this condition reduces to $5760 N-$ $1856 a>0$, hence it always hold when $N \geq 1$ and $a \leq 1$. It follows that $D_{N}<S_{N-1}$.

To show $D_{N}>S_{N}$, we need to show

$$
\frac{8 N a-15 a^{2}+3 a \sqrt{64 N^{2}-48 N a+25 a^{2}}}{128 N^{3}}>\frac{a}{4 N(N+1)}-\frac{a^{2}(2 N+1)}{24 N^{2}(N+1)^{2}}
$$


which holds whenever

$$
16 N[6 N(N+1)-a(2 N+1)]<3(N+1)^{2}\left(8 N-15 a+3 \sqrt{64 N^{2}-48 N a+25 a^{2}}\right) .
$$

Simplifying and squaring, we see that this expression holds when

$$
\left(45 a-24 N+13 N^{2} a+74 N a-48 N^{2}+72 N^{3}\right)^{2}<81(N+1)^{4}\left(64 N^{2}-48 N a+25 a^{2}\right)
$$

If we define the polynomial

$$
\begin{aligned}
P(a, N)= & -\left(58 N^{3}+193 N^{2}+172 N+45\right) a^{2} \\
& +\left(180 N^{4}+858 N^{3}+1134 N^{2}+510 N+54\right) a \\
& -432 N^{4}-1008 N^{3}-720 N^{2}-144 N
\end{aligned}
$$

expression (21) holds whenever $32 N P(a, N)<0$, so we want to show that $P(a, N)<0$ for all $a \in[0,1]$ and all $N \geq 1$.

For any $N, P$ is maximized at $\hat{a}=\frac{180 N^{4}+858 N^{3}+1134 N^{2}+510 N+54}{116 N^{3}+386 N^{2}+344 N+90}$. As $\hat{a}>1$ for all $N \geq 1$ it follows that $P(a, N)<P(1, N)$ for all $a<1$. As $P(1, N)=-252 N^{4}-208 N^{3}+221 N^{2}+$ $194 N+9<0$ for all $N \geq 1$, expression (21) holds for all $a \in[0,1]$ and all $N \geq 1$.

\section{A.6. Proof of Proposition 9}

It suffices to show that $a m+\frac{S_{N}}{\Psi_{N}} \leq a\left(m-\frac{1}{4 N}\right)+\frac{S_{N}}{\Psi_{2 N}}$. To see this, it follows from (20) that

$$
\frac{C}{\Psi_{N}}=\frac{1}{2}\left(1-\frac{a}{4 N}\right)+\sqrt{\left[\frac{1}{2}\left(1-\frac{a}{4 N}\right)\right]^{2}-N C}
$$

Combining this with Lemma 1, we get

$$
\begin{aligned}
& a m=\left(\frac{1}{2}+\frac{a}{8 N}-\sqrt{\left.\left(\frac{1}{2}-\frac{a}{8 N}\right)^{2}-N C\right)}\right. \\
&-\sqrt{\left(\frac{1}{2}+\frac{a}{8 N}-\sqrt{\left.\left(\frac{1}{2}-\frac{a}{8 N}\right)^{2}-N C\right)^{2}-\frac{a}{2 N}\left(\frac{1}{2}+\frac{a}{8 N}-\sqrt{\left(\frac{1}{2}-\frac{a}{8 N}\right)^{2}-N C}-\frac{a}{4 N}\right)}\right.}
\end{aligned}
$$

so

$$
\begin{aligned}
2 a m=1 & +\frac{a}{4 N}-2 \sqrt{\left(\frac{1}{2}-\frac{a}{8 N}\right)^{2}-N C} \\
& -2 \sqrt{\frac{1}{2}+\frac{3 a^{2}}{32 N^{2}}-2\left(\frac{1}{2}-\frac{a}{8 N}\right) \sqrt{\left(\frac{1}{2}-\frac{a}{8 N}\right)^{2}-N C}-N C}
\end{aligned}
$$


Using (22) in (19) and inserting $S_{N}=\frac{a}{4 N(N+1)}-\frac{a^{2}(2 N+1)}{24 N^{2}(N+1)^{2}}$, we get that the expression holds whenever

$$
\begin{aligned}
Q(a, N)= & \frac{\sqrt{A(a, N)}}{\sqrt{768 N(N+1)}+\frac{\sqrt{B(a, N)}}{\sqrt{192} N(N+1)}} \\
& +\sqrt{\frac{D(a, N)}{24 N^{2}(N+1)^{2}}-\left(1-\frac{a}{4 N}\right) \frac{\sqrt{B(a, N)}}{\sqrt{12} N(N+1)}}+\frac{a}{16 N}-1 \geq 0
\end{aligned}
$$

with

$$
\begin{aligned}
& A(a, N)=192 N^{4}-432 N^{3} a+384 N^{3}+131 N^{2} a^{2}-480 N^{2} a+192 N^{2}+70 N a^{2}-48 N a+3 a^{2} \\
& B(a, N)=48 N^{4}-72 N^{3} a+96 N^{3}+19 N^{2} a^{2}-96 N^{2} a+48 N^{2}+14 N a^{2}-24 N a+3 a^{2} \\
& D(a, N)=48 N^{4}-24 N^{3} a+96 N^{3}+17 N^{2} a^{2}-24 N^{2} a+48 N^{2}+22 N a^{2}+9 a^{2}
\end{aligned}
$$

It is seen that $Q(0, N)=0$. Also for all $N>1, Q$ is increasing in $a$, so for all $a \geq 0$ and $N>1$ we have $Q(a, N) \geq 0$. Finally, one can verify that $Q(a, 1)$ is minimized at $a=0$ yielding $Q(0,1)=0$. Hence for all $a \in(0,1)$ and $N \geq 1$ we have $Q(a, N) \geq 0$ so $S_{N} \leq D_{N}$

\section{References}

Alesina, A., and Spolaore, E. (1997). "On the number and size of nations." Quarterly Journal of Economics, 112(4), 1027-1056.

Alesina, A., and Spolaore, E. (2003). The Size of Nations. The MIT Press.

Bergstrom, T., Blume, L., and Varian, H. (1986). "On the private provision of public goods." Journal of Public Economics, 29, 25-49.

Bogomolnaia, A., Le Breton, M., Savvateev, A., and Weber, S. (2007). "Stability under unanimous consent, free mobility and core." International Journal of Game Theory, $35,185-204$.

Bogomolnaia, A., Le Breton, M., Savvateev, A., and Weber, S. (2008a). "Heterogeneity gap in stable jurisdiction structures." Journal of Public Economic Theory, 10, 455-473.

Bogomolnaia, A., Le Breton, M., Savvateev, A., and Weber, S. (2008b). "Stability of jurisdiction structures under the equal share and median rules." Economic Theory, 34, $525-543$.

Bolton, P., and Roland, G. (1997). "The breakup of nations: A political economy analysis." Quarterly Journal of Economics, 112(4), 1057-1090.

Buchanan, J. M. (1965). "An economic theory of clubs." Economica, 32, 1-14.

Casella, A., and Feinstein, J. S. (2002). "Public goods in trade: On the formation of markets and jurisdictions." International Economic Review, 43, 437 - 462.

Cornes, R., and Sandler, T. (1996). The Theory of Externalities, Public Goods, and Club Goods. Cambridge: Cambridge University Press. 
Cremer, H., De Kerchove, A.-M., and Thisse, J.-F. (1985). "An economic theory of public facilities in space." Mathematical Social Sciences, 9(3), 249 - 262.

Drèze, J., Le Breton, M., Savvateev, A., and Weber, S. (2008). "Almost" subsidy-free spatial pricing in a multi-dimensional setting." Journal of Economic Theory, 143(1), $275-291$.

Drèze, J., Le Breton, M., and Weber, S. (2007). "Rawlsian pricing of access to public facilities: A unidimensional illustration." Journal of Economic Theory, 136(1), 759766.

Ellickson, B., Grodal, B., Scotchmer, S., and Zame, W. (2001). "Clubs and the market: Large finite economies." Journal of Economic Theory, 101, 40-77.

Ellickson, B., Grodal, B., Scotchmer, S., and Zame, W. R. (1999). "Clubs and the market." Econometrica, 67(5), 1185-1217.

Ellickson, B., Grodal, B., Scotchmer, S., and Zame, W. R. (2006). "The organization of production, consumption and learning." In C. Schultz, and K. Vind (Eds.), Institutions, Equilibria and Efficiency, chap. 9, 149-185, Berlin: Springer.

Evers, A., and Laville, J.-L. (2004). The Third Sector in Europe. Cheltenham: Edward Elgar.

Fujita, M., and Thisse, J.-F. (2002). Economics of Agglomeration. Cities, Industrial Location, and Regional Growth. Cambridge: Cambridge University Press.

Fukuyama, F. (2011). The Origins of Political Order: From Prehuman Times to the French Revolution. London: Profile Books.

Haimanko, O., Le Breton, M., and Weber, S. (2004). "Voluntary formation of communities for the provision of public projects." Journal of Economic Theory, 115, 1-34.

Haimanko, O., Le Breton, M., and Weber, S. (2005). "Transfers in a polarized country: Bridging the gap between efficiency and stability." Journal of Public Economics, 89(7), 1277-1303.

Hotelling, H. (1929). "Stability in competition." The Economic Journal, 39, 41-57.

Jehiel, P., and Scotchmer, S. (1997). "Free mobility and the optimal number of jurisdictions." Annales d'Economie et de Statistique, 45, 219-31.

Jehiel, P., and Scotchmer, S. (2001). "Constitutional rules of exclusion in jurisdiction formation." Review of Economic Studies, 68, 393 - 413.

Krugman, P. (1993). "On the number and location of cities." European Economic Review, $37(2-3), 293-298$.

Le Breton, M., and Weber, S. (2003). "The art of making everybody happy: How to prevent a secession." IMF Staff Papers, 50, 403-35.

Le Breton, M., Weber, S., and Drèze, J. (2005). "Secession-proofness in large heterogeneous societies." Économie publique, 17(2), 197-220. 
McGann, A. J. (2002). "The advantages of ideological cohesion: A model of constituency representation and electoral competition in multi-party democracies." Journal of Theoretical Politics, 14, 37-70.

Milchtaich, I., and Winter, E. (2002). "Stability and segregation in group formation." Games and Economic Behavior, 38(2), 318 - 346.

Musatov, D., Savvateev, A., and Weber, S. (2015). "Gale-Nikaido-Debreu and MilgromShannon: Market interactions with endogenous community structures.", CEPR Discussion Paper 10641.

Paúly, M. V. (1967). "Clubs, commonality, and the core: An integration of game theory and the theory of public goods." Economica, 34(135), pp. 314-324.

Ruta, M. (2005). "Economic theories of political (dis)integration." Journal of Economic Surveys, 19, 1-21.

Salop, S. C. (1979). "Monopolistic competition with outside goods." Bell Journal of Economics, 10(1), 141-156.

Scotchmer, S. (2002). "Local public goods and clubs." In A. J. Auerbach, and M. Feldstein (Eds.), Handbook of Public Economics, vol. 4, 1997-2042, Amsterdam: Elsevier.

Tabuchi, T., Thisse, J.-F., and Zeng, D.-Z. (2005). "On the number and size of cities." Journal of Economic Geography, 5, 423-48.

Tiebout, C. M. (1956). "A pure theory of local expenditures." Journal of Political Economy, 64(5), 416-424.

Weber, S., Makarov, V. L., and Savvateev, A. V. (2014). "An equilibrium model with mixed federal structures." Bulletin of Irkutsk State University. Series Mathematics, 8, $62-70$.

Westhoff, F. (1977). "Existence of equilibria in economies with a local public good." Journal of Economic Theory, 14(1), 84 - 112.

Wooders, M. (1987). "Stability of jurisdiction structures in economics with local public goods." Mathematical Social Sciences, 15, 29-49.

Wooders, M. (1989). "A Tiebout theorem." Mathematical Social Sciences, 18, 33-55.

Wooders, M. (2012). "The theory of clubs and competitive coalitions." Annual Review of Economics, 4(1), 595-626. 